APJ, IN PRESS

Preprint typeset using $\mathrm{LAT}_{\mathrm{E}} \mathrm{X}$ style emulateapj v. 5/2/11

\title{
WHAT ARE LITTLE WORLDS MADE OF? STELLAR ABUNDANCES AND THE BUILDING BLOCKS OF PLANETS
}

\author{
ERIC GaIdos ${ }^{1}$ \\ Department of Geology \& Geophysics, University of Hawai'i at Mānoa, Honolulu, HI 96822 \\ ApJ, in press
}

\begin{abstract}
If the photospheres of solar-type stars represent the composition of circumstellar disks from which any planets formed, spectroscopic determinations of stellar elemental abundances offer information on the composition of those planets, including smaller, rocky planets. In particular, the $\mathrm{C} / \mathrm{O}$ ratio is proposed to be a key determinant of the composition of solids that condense from disk gas and are incorporated into planets. Also, planets may leave chemical signatures on the photospheres of their host stars by sequestering heavy elements, or by being accreted by the stars. The presence, absence, and composition of planets could be revealed by small differences in the relative abundances between stars. I critically examine these scenarios and show that (i) a model of Galactic chemical evolution predicts that the $\mathrm{C} / \mathrm{O}$ ratio is expected to be close to the solar value and vary little between dwarf stars in the solar neighborhood; (ii) spectroscopic surveys of $\mathrm{M}$ dwarf stars limit the occurrence of stars with $\mathrm{C} / \mathrm{O} \gtrsim 1$ to $<10^{-3}$; and (iii) planetesimal chemistry will be controlled by the composition of oxygen-rich dust inherited from the molecular cloud and processed in a dust-rich environment, not a gas with the stellar composition. A second generation of more reduced planetesimals could be produced by re-equilibration of material with dust-depleted gas. Finally, I discuss how minor differences in relative abundances between stars that correlate with condensation temperature can be explained by dust-gas segregation, perhaps in circumstellar disks, rather than planet formation.
\end{abstract}

Subject headings: stars:abundances - planets and satellites:gaseous planets - planets and satellites:formation - methods:spectroscopic

\section{INTRODUCTION}

Ground-based Doppler radial velocity surveys and space-based photometric surveys have established that planets are very common, and perhaps ubiquitous, around Sun-like stars. In particular, analysis of detections by the NASA Kepler mission show that planets with radii of 1-2 Earth radii $\left(\mathrm{R}_{\oplus}\right)$ are far more common than larger planets (Petigura et al. 2013; Silburt et al. 2014). Comparisons between estimates or upper limits on mass from Doppler radial velocity measurements and radii from Kepler also suggest that the densities of planets (at least planets on short-period orbts) smaller than $1.5 \mathrm{M}_{\oplus}$ are consistent with a "rocky" (silicates + metals) composition, while larger planets have an additional lowmolecular weight (e.g. hydrogen) envelope (Marcy et al. 2014). However, the data are insufficiently precise to inform as to the exact composition of these planets and regardless there are degeneracies in mass and radius with different bulk compositions.

The photospheres of dwarf stars on the main sequence are broadly representative of the material from which the star and any circumstellar disk formed. Additional information about planet composition might therefore be inferred by determining elemental abundances in the hoststar photosphere. This connection is supported by the observation that the abundances of refractory elements in the solar photosphere are, to a large extent, mirrored by that in primitive chondritic meteorites, the latter widely used as an analog of the primordial building blocks of

\footnotetext{
gaidos@hawaii.edu

${ }^{1}$ Visiting Researcher, Max Planck Institut für Astronomie, Heidelberg, Germany
}

planets (e.g., Lodders 2003). To a lesser extent, these abundances are reflected in the bulk composition of the planets such as Earth, but with some important exceptions.

The two most abundant heavy elements in the Galaxy and Sun (and presumably planet-forming disks) are carbon $(\mathrm{C})$ and oxygen $(\mathrm{O})$. In the cool interstellar medium (ISM), star-forming regions, and molecular cloud cores, $\mathrm{C}$ and $\mathrm{O}$ are present primarily as the $\mathrm{CO}$ molecule (e.g., Bolatto et al. 2013). Because of the unit stochiometry of the $\mathrm{CO}$ molecule, whether $\mathrm{C}$ or $\mathrm{O}$ is in excess controls which element is available to form other compounds and thus the chemistry of the gas and solids that condense or equilibrate with the gas. The solar ratio of these two elements is presently estimated at $0.55 \pm 0.12$ (Caffau et al. 2011, see also Asplund et al. 2009), however this value is not necessarily universal. The two elements differ in their predicted pathways of stellar nucleosynthesis and $\mathrm{C} / \mathrm{O}$ has a strong positive correlation with metallicity (Teske et al. 2014; Nissen et al. 2014).

Motivated by condensation scenarios for the solar nebula (Barshay \& Lewis 1976) and observations of widely varying $\mathrm{C} / \mathrm{O}$ among solar-type stars (Edvardsson et al. 1993), Gaidos (2000) proposed that $\mathrm{C} / \mathrm{O}$ controls the abundance of water in planetary systems. During condensation from a hot gas with $\mathrm{C} / \mathrm{O} \ll 1$, excess oxygen forms refractory silicate minerals and eventually $\mathrm{H}_{2} \mathrm{O}$. However, in systems with $\mathrm{C} / \mathrm{O}$ near a critical value (about 0.88), no $\mathrm{O}$ for water is available after silicate condensation and planets accrete from "dry" material. At still higher $\mathrm{C} / \mathrm{O}$, the deficiency in $\mathrm{O}$ causes carbides and graphite to become more stable and replace silicates in the condensation sequence; thus "carbide planets" with 
very un-Earth-like mineralogies, interior structures, and atmospheres might form around stars with $\mathrm{C} / \mathrm{O} \gtrsim 1$.

Many analyses of the spectra of solar-type stars obtained for galactic chemical evolution (GCE) studies (Gustafsson et al. 1999) and Doppler radial velocity surveys found that a significant fraction have $\mathrm{C} / \mathrm{O} \geq 1$ (Ecuvillon et al. 2004, 2006; Delgado Mena et al. 2010; Petigura \& Marcy 2011). A series of subsequent works have predicted the final bulk composition of planets by modeling (i) the sequential condensation of elements and formation of planetesimals from a cooling gas disk with an initial composition set by host star abundances; and (ii) the accretion of those planetesimals into planets via integration of the dynamical equations of motion. These scenarios have been applied to specific systems where the photospheric abundances of elements have been estimated, variously predicting water-rich "ocean planets" and "carbon planets" lacking any water (Bond et al. 2010; Elser et al. 2012; Carter-Bond et al. 2012; Moriarty et al. 2014).

One question at the foundation of these works is whether $\mathrm{C} / \mathrm{O}$ really varies widely among Galactic disk stars in the solar neighborhood and ever approaches unity. Abundances of $\mathrm{C}$ and $\mathrm{O}$ are more uncertain than for many other elements because of the limited number of usable absorption lines, confusion with lines of other elements, and non-LTE corrections. Recent studies have revised the $\mathrm{C} / \mathrm{O}$ of nearby solar-type stars, including those that host known planets, downwards (Nissen 2013; Teske et al. 2013a,b, 2014). These newer studies find no cases where $\mathrm{C} / \mathrm{O}>0.8$, the threshold where carbide minerals are expected to first form. Fortney (2012) has pointed out that the occurrence of C-rich systems in surveys of solar-type stars conflicts with the lack of known C-rich ultracool $\mathrm{T}$ dwarfs, as the latter are easily distinguished by their spectra (Fortney 2012).

A second issue is the assumption that the solids in planetary systems condense from a cooling gas of stellar composition in a circumstellar disk. Equilibrium condensation can explain some solids in primitive chondrites, widely considered analogs to the building blocks of planets, but these solids constitute only a small fraction of those meteorites. Calcium aluminum inclusions (CAIs) and amoeboid olivene agregates, the most abundant refractory condensates in chondritic meteorites, have volume abundances $<10 \%$ and typically $<1 \%$ ( $\operatorname{Scott} \mid 2007$; Hezel et al. 2008). The dominant constituents of chondritic meteorites are chondrules and fine-grained matrix, and there is compelling evidence that these are the product of incomplete sublimation and chemical alteration of pre-existing solids, e.g. older generations of solids and even pre-solar dust from the parent molecular cloud (Huss et al. 2003; Trinquier et al. 2009; Burkhardt et al. 2012). This processing occurred under conditions that were very different from a gas of solar composition, i.e. an oxygen-rich environment and dust-to-gas ratios $\geq 1$ (Alexander \& Ebel 2012). This environment could have been created by gravitational settling to the mid-plane of the protoplanetary disk. Because of this, the composition of planeteismals reflects the composition of dust grains in the molecular clouds cores that collapse to form stars and planets, plus subsequent thermal processing at midplane conditions. ISM dust was, in turn, the product of processing in the interstellar medium involving cycling of order $10^{2}$ times between denser, cooler clouds, where it accreted mantles of atoms, and the hotter intercloud phase, where shock-heated ions and high-energy radiation (UV, X-rays, cosmic rays) sputtered atoms from the grains (Tielens 2012a).

An equally intriguing possibility is that the compositions of planetesimals and/or planets can be inferred from differential measurements of abundances in the photospheres of stars. This approach is motivated by the detection of small differences in stellar abundances of elements that are more refractory (higher condensation temperature $T_{c}$ ) and thus more likely to be incorporated into planets (e.g., Meléndez et al. 2009). These differences could arise either from the accretion of planetesimals or planets onto the star, after the dissipation of disk gas, or the sequestration of solids into planets, and accretion of the dust-poor gas onto the star 2 This leads to a prediction that these differences correlate with the presence or properties of planetary systems, and provides a potential "short-cut" to discovering planets as well as estimating their chemical composition. However, subsequent analyses of larger samples of stars does not support such a correlation (González Hernández et al. 2013; Liu et al. 2014), and alternative explanations for these differences should be considered.

In this work, I critically examine the hypothesis that $\mathrm{C} / \mathrm{O}$ varies significantly among neighboring stars in the Galactic disk (and hence the host stars of Dopplerdetected exoplanets) from both theoretical (Sec. 2), observational (Sec. 3), and cosmochemical (Sec. 4) perspectives. For the first I develop a GCE model to predict the abundance of $\mathrm{C}$ and $\mathrm{O}$ over the history of the Galactic disk in the vicinity of the Sun. For the second, I use the spectra obtained in $\mathrm{M}$ dwarf surveys to place strict upper limits on the occurrence of C-rich single M dwarfs among recent large surveys. For the third, I combine a simple model of interestellar dust evolution with UV measurements of the depletion of heavy elements in the ISM to determine what controls the $\mathrm{C} / \mathrm{O}$ ratio and by how much it may vary. Finally, I develop an alternative explanation that can explain the observed elemental relative abundance differences between solar-type stars, without resorting to possible signatures of planet formation (Sec. 5).

\section{EXPECTATIONS FOR C/O FROM GALACTIC} CHEMICAL EVOLUTION

Galactic chemical evolution (GCE) models predict changes in the abundances of elements and isotopes with time in both stars and the ISM (Prantzos 2008). They combine yields of nucleosynthetic products in stellar winds and ejecta with the stellar initial mass function (IMF) and a prescription for star formation rate to estimate the production of elements and isotopes. These are integrated with descriptions of the flow of heavy elements back into the ISM and subsequent incorporation in longlived low-mass stars and remnants from high-mass stars. Production varies with both stellar progenitor mass and metallicity, thus the changing metallicity of new generations of stars must be tracked in a GCE.

2 This was one explanation for the well-established correlation between overall metallicity and giant planets (Gonzalez 1998; Santos et al. 2004; Fischer \& Valenti 2005) 
Carbon and oxygen are synthesized in stars more massive than $1.5 \mathrm{M}_{\odot}$ and injected into the interstellar medium through winds and Type II supernovae $(\mathrm{SN}):$ Type I SN are predicted to contribute $<0.3 \%$ (Gehrz et al. 1998). GCE models generally predict a positive trend of $\mathrm{C} / \mathrm{O}$ with metallicity (and hence time) as well as variation between stellar populations with different chemical histories (e.g., Tinsley 1980; Timmes et al. 1995; Cescutti et al. 2009). These trends are broadly observed within disk stars and between the thin, thick-disk and bulge populations, and even between massive and metal-poor irregular dwarf galaxies (Garnett et al. 1995; Cescutti et al. 2009; Nissen et al. 2014; Esteban et al. 2014). At least two effects are thought to contribute to this correlation: (i) the short main-sequence lifetime of massive stars, which are major contributors to $\alpha$ elements including $\mathrm{C}$ and $\mathrm{O}$, compared to intermediate mass asymptotic giant branch (AGB) progenitors, which contribute comparatively more to the $\mathrm{C}$ budget 3 ; (ii) increased mass loss and $\mathrm{C}$ yield from younger, more metalrich AGB stars at the expense of O. Mass-loss rates depends on metallicity through its effect on the opacity of outer stellar atmospheres and the impact on their structure. The implications of the abundance dependence of $\mathrm{C} / \mathrm{O}$ yields for the chemical evolution of galaxies has been previously pointed out (e.g., Maeder 1992; Frayer \& Brown 1997).

The $\mathrm{C} / \mathrm{O}$ of bulge and disk populations diverge due to a combination of differences in the stellar IMF, winddriven mass loss in the former and the accretion of primordial metal-poor gas in the former. The flatter IMF of bulge stars implies a higher relative production of $\mathrm{O}$ from more abundant massive stars. Addition (loss) of mass from a star-forming system will increase (decrease) the amount of star formation required to arrive at a given metal abundance and therefore the $\mathrm{C} / \mathrm{O}$ at that abundance.

One feature of the statistics of stellar metallicities of the Galactic disk in the neighborhood of the Sun is the relatively narrow dispersion and paucity of metal-poor stars. This is often called the "G dwarf problem" and translates into a flat age-metallicity relation for the most of the history of the Galactic disk, at least in the solar neighborhood. It is actually not a problem for GCE models if addition of metal-poor gas to the disk is admitted to balance stellar nucleosynthesis (e.g., Holmberg et al. 2007). If $\mathrm{C} / \mathrm{O}$ is strongly correlated with metallicity then a flat age-metallicity relation might mean that $\mathrm{C} / \mathrm{O}$ evolves very little (e.g., Wheeler et al. 1989).

To re-visit the question of the evolution of $\mathrm{C} / \mathrm{O}$ in the solar neighborhood, the abundances of the two elements were calculated on an isotope-by-isotope basis using the model described in detail in the Appendix. The model simulates the production of these isotopes and release into the ISM in SN explosions, winds from massive stars and AGB stars, sequestration of isotopes into long-lived low-mass stars, and addition of metal-poor gas by infall onto the Galactic disk. The ISM is described by two components; an inter-cloud medium which produces molecu-

${ }^{3}$ While O yields are relatively insensitive to model parameters and are consistent from model to model (Woosley \& Weaver 1995), the $\mathrm{C}$ yield of intermediate-mass stars depend sensitively on the amount of convective "dredge-up" (Renzini \& Voli 1981). lar clouds but no stars, and a star-forming giant molecular cloud component. Yields for intermediate- and massive stars are taken from a variety of recent sources. Bestfit parameters describing the timescale of exponentiallydeclining gas infall on the disk, the initial metallicity of the infalling gas, the age of the stellar population at the solar galactocentric radius, the index of the power-law describing the IMF for massive stars, and the power-law relationship between star formation rate and gas surface density were found by a Monte-Carlo Markov Chain analysis: The observational constraints were the age of the Sun, the present mass surface density of stars, stellar remanants, and gas at the solar galactocentric radius, solar metallicity (here taken to be $\mathrm{C}+\mathrm{O}$ ), the current metallicity of the ISM, and its intrinsic standard deviation.

The prediction evolution of $\mathrm{C} / \mathrm{O}$ is compared to the solar value from Caffau et al. (2011) in Fig. 1. After initial transients that die away after a few hundred Myr, there is only moderate evolution in $\mathrm{C} / \mathrm{O}$ during most of the history of the Galactic disk. The $\mathrm{C} / \mathrm{O}$ ratio rises from 0.5 to about 0.65 over the first $6 \mathrm{Gyr}$ in response to increasing input from stars of lower mass and higher metallicity, as described above, and declines slightly thereafter. The predicted value at the time of the Sun's formation is 0.64 , withinin $1 \sigma$ of the Caffau et al. (2011) value. The formal error in the predicted value, based on the standard deviation of the MCMC chain after removal of the "burn in", is only 0.003 , but this value does not reflect the dominant source of uncertainty in these calculations - the nucleosynthetic yields. Although the model is unable to exactly reproduce the solar values of $\mathrm{C}$ and $\mathrm{O}$ abundances, correctly reproducing these using GCE been always been challenging. Maeder (1992) also found that satisfactorily reproducing a "flat" $\mathrm{C} / \mathrm{O}$ places constraints on the remnant or "cut-off" mass at the center of a SN progenitor which is not injected into the ISM.

Figure 2 plots the predicted $\mathrm{C} / \mathrm{O}$ evolution vs. predicted $[\mathrm{O} / \mathrm{H}]$, again compared to the Sun. Also plotted are the values found by Nissen et al. (2014) for Galactic disk stars (filled points) and bulge or halo stars (open points) and converted to absolute values assuming a so$\operatorname{lar} \mathrm{C} / \mathrm{O}$ of 0.55 . The predicted trend is perfectly consistent with the disk values if an offset of about $16 \%$ with the predicted vs. measured Solar value is artificially removed. The absence of data for disk stars at low $[\mathrm{O} / \mathrm{H}]$ is a consequence of the age-metallicity relationship: comparatively little time elapsed $(<1$ Gyr according to the model) and few stars formed in this interval.

The predicted constancy of $\mathrm{C} / \mathrm{O}$ contrasts with previous predictions for an increase in $\mathrm{C} / \mathrm{O}$ with metallicity/time. For example, both Cescutti et al. (2009) and Mattsson (2010) predict a positive slope of $\sim 1 \mathrm{dex} / \mathrm{dex}$ in $[\mathrm{C} / \mathrm{O}]$ vs. $[\mathrm{O} / \mathrm{H}]$, and thus a $\mathrm{C} / \mathrm{O}$ of about 0.06 at $[\mathrm{O} / \mathrm{H}]=-1$. Since the model and the best-fit parameter values presented here are similar to those of previous works the most likely cause of this difference is in the particular nucleosynthetic yields that were used. The yields from AGB stars have been significantly revised (Karakas 2010) but in the model these are minority contributors to the $\mathrm{C}$ and $\mathrm{O}$ budgets. Indeed, if there contribution is completely removed, $\mathrm{C} / \mathrm{O}$ still changes little and the predicted solar $\mathrm{C} / \mathrm{O}$ is slightly closer to the Caffau et al. (2011) value. Instead, the predictions of this model probably stand out from previous results because of the dom- 


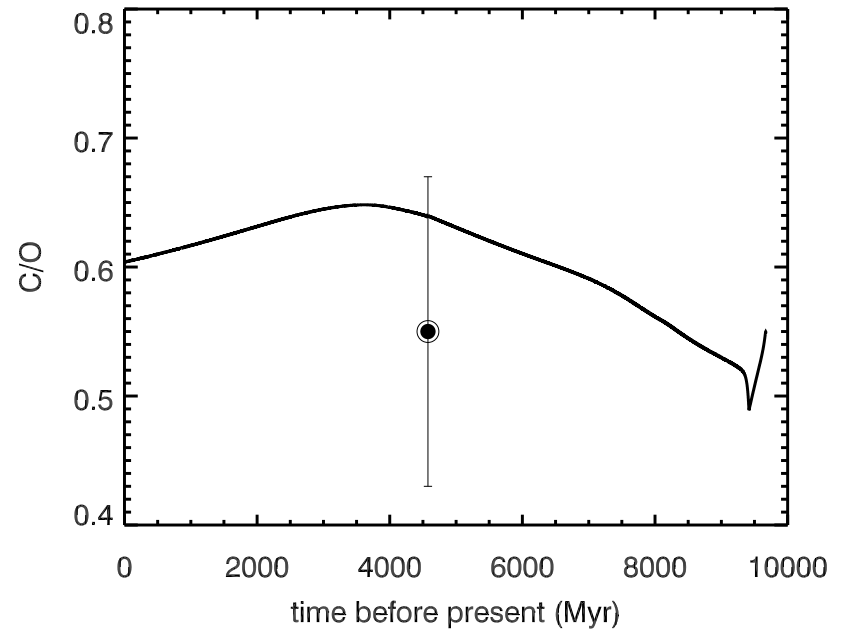

Figure 1. Evolution of $\mathrm{C} / \mathrm{O}$ at the solar galactocentric radius according to the GCE model described in the text and Appendix. The solar value and its uncertainty from Caffau et al. (2011) is plotted.

inant contribution of massive stars to both $\mathrm{C}$ and $\mathrm{O}$. Since these stars have very short lives compared to the chemical evolution of the disk, there is no effect from delayed contribution, and the $\mathrm{C} / \mathrm{O}$ rapidly approaches a steady-state value.

Previous works have pointed to favorable comparisons of model predictions with observations, but the latter are combined data on bulge, halo, and thick- and thindisk populations, therefore assuming a common origin for these populations that may not exist. For example, the $[\mathrm{C} / \mathrm{O}]$ of thin disk stars presented by Bensby \& Feltzing (2008) and Cescutti et al. (2009) show no correlation with $[\mathrm{O} / \mathrm{H}]$ and it is only when bulge and thick disk stars are added that such a correlation appears. The apparent consistency between the predicted and observed trends of $[\mathrm{C} / \mathrm{O}$ ] vs. [O/H] (Cescutti et al. 2009; Mattsson 2010) belies the fact that the models were tuned for the (thin) disk population in the solar neighborhood and should not be compared with other stellar populations.

It is indisputable that metal-poor bulge/halo stars have low $\mathrm{C} / \mathrm{O}$ compared to the solar neighborhood (Fabbian et al. 2009; Nissen et al. 2014). The model presented here cannot, and was not designed to, explain these populations, but their abundances might be a result of a flatter IMF, early loss of gas and/or truncation of star formation. But bulge/halo stars are rare in the solar neighborhood and because their lines are very weak they are usually avoided by planet searches using the Doppler radial velocity method.

$\mathrm{C} / \mathrm{O}$ may also vary at some level within the solar galactocentric annulus because the sources of nuclides (stars and stellar clusters) are discrete and mixing by rotational shear and epicyclic dynamics is not completely efficient. However, GCE is occurring on a timescale much longer (few Gyr) than the rotational time of the Galaxy at $8 \mathrm{kpc}$ ( $\sim 250 \mathrm{Myr}$ ) which governs the rate of mixing, and thus this limits the magnitude of such heterogeneities.

\section{M DWARFS AS PROBES OF HIGH C/O}

In solar-type stars, the absorption lines of $\mathrm{C}$ and $\mathrm{O}$ are weak and $\mathrm{C} / \mathrm{O}$ has little effect on the overall spec-

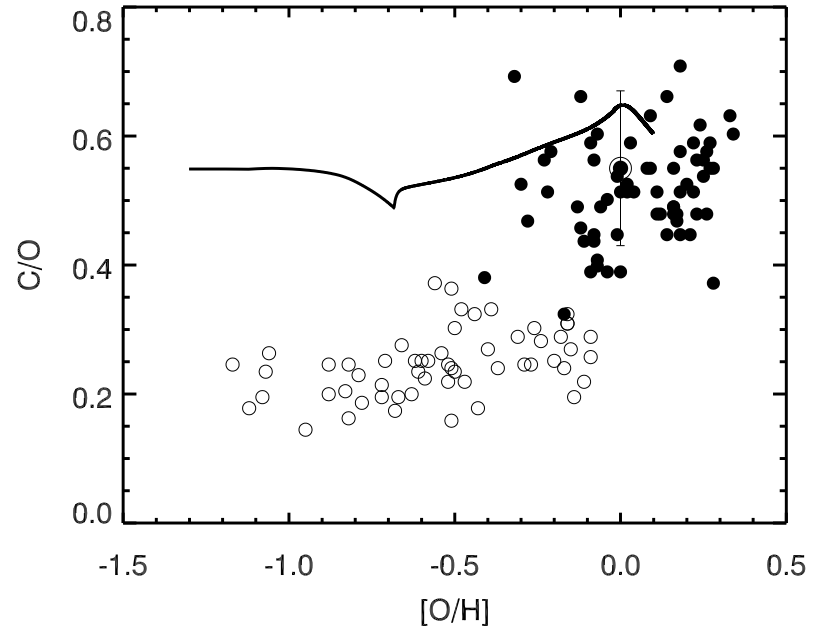

Figure 2. Evolution of $\mathrm{C} / \mathrm{O}$ at the solar galactocentric radius according to the GCE model described in the text and Appendix, plotted vs. [O/H]. The Caffau et al. (2011) solar value is plotted as well as values for Galactic disk stars (filled points) and bulge/halo stars (open points) from Nissen et al. (2014) and tied to the solar value.

trum. Fortney (2012) pointed out that cooler stars, i.e. brown dwarfs, offer more obvious constraints on the occurrence of high $\mathrm{C} / \mathrm{O}$ systems. The spectra of $\mathrm{M}$ dwarfs, with effective temperatures $T_{\text {eff }}<3900 \mathrm{~K}$, also include absorption bands of O-containing molecules, e.g. $\mathrm{TiO}$, $\mathrm{VO}$, and $\mathrm{CaOH}$, which are sensitive to the available $\mathrm{O}$ abundance (Schmidt et al. 2009), and hence C/O. At $\mathrm{C} / \mathrm{O} \geq 1, \mathrm{TiO}$ is absent and the Schwan bands of $\mathrm{C}_{2}$, $\mathrm{CN}$, and $\mathrm{CH}$ should appear. Such spectra are characteristic of carbon stars, evolved stars in which dredge-up of carbon-righ interiors has occurred (AGB stars). If the initial mass function of star formation is chemically invariant, a limit on the occurrence of $\mathrm{C}$-rich $\mathrm{M}$ dwarfs is also a limit on C-rich $\mathrm{G}$ dwarfs.

Dwarf carbon (dC) star have been identified (Dahn et al. 1977) but these probably accreted carbonrich gas from a present or former evolved companion (Behara et al. 2010; Green 2013). Overall, dC stars are uncommon (Downes et al. 2004; Green 2013). Carbon enhanced metal-poor (CEMP) stars in the Galactic halo appear are also rare overall but more prevalent among (initially) metal-poor systems because less accreted $\mathrm{C}$ is required to increase $\mathrm{C} / \mathrm{O}$ (Beers et al. 1992). Any tally of intrinsically carbon-rich dwarfs must remove these interlopers or be considered an upper limit.

I considered three spectral indices which are continuum-normalized measures of the emission in specific bands (Reid et al. 1995): $\mathrm{CaH}$, which is the mean

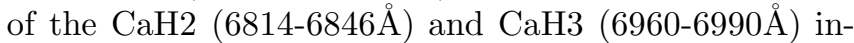

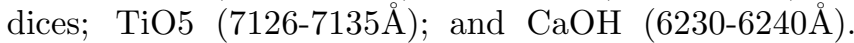
Values of these temperature- and gravity-sensitive indices are highly and positively correlated among solarmetallicity $\mathrm{M}$ dwarfs. Carbon-rich stars can be identified by relatively weak bands of oxygen-containing $\mathrm{TiO}$ and $\mathrm{CaOH}$, i.e. high $\mathrm{TiO} 5$ or $\mathrm{CaOH}$ indices, for a given strength of the non-oxygen-containing $\mathrm{CaH}$. However, indices of metal-poor subdwarfs or "extreme" subdwarfs exhibit similar behavior (Jao et al. 2008; Woolf et al. 2009; Raipurohit et al. 2014). 


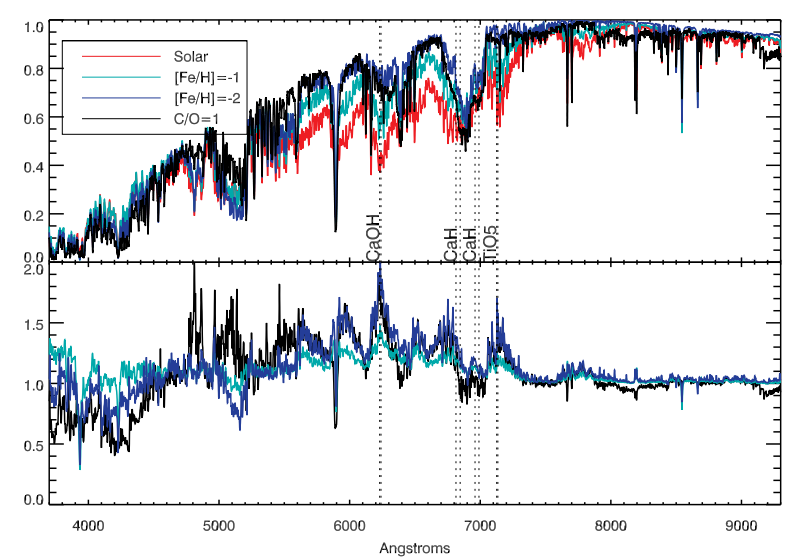

Figure 3. Top: Normalized PHOENIX BT-Settl synthetic spectra of M dwarfs with $T_{\text {eff }}=3700 \mathrm{~K}, \log g=5$, and solar abundances (Caffau et al. 2011, red line), metal-poor subdwarf $([\mathrm{Fe} / \mathrm{H}]=-1$, $[\alpha / \mathrm{Fe}]=0$, green line $)$, extreme subdwarf $([\mathrm{Fe} / \mathrm{H}]=-2,[\alpha / \mathrm{Fe}]=$ $+0.4, \log g=5.5$, blue line), and solar-metallicity star except some $\mathrm{O}$ as $\mathrm{C}$ such that $\mathrm{C} / \mathrm{O}=1$ (red line). Bottom: ratios of the three non solar-metallicity spectra described above to the solar metallicity spectrum.

To guide discrimination between $\mathrm{C}$-rich $\mathrm{M}$ dwarfs and metal-poor stars based on indices, synthetic spectra were generated using the BT-Settl version of the PHOENIX models (Allard et al. 2011). Four cases were considered: a solar-metallicity star, a star with $[\mathrm{M} / \mathrm{H}]=0$ but $\mathrm{C}$ and $\mathrm{O}$ adjusted so that $\mathrm{C} / \mathrm{O}=1$, a metal-poor subdwarf (sd) with $[\mathrm{M} / \mathrm{H}]=-1$, and an extreme subdwarf (esd) with $[\mathrm{Fe} / \mathrm{H}]=-2$. The last two cases also had an alphaelement enhancement $[\alpha / \mathrm{Fe}]=+0.4$. Effective temperatures over the entire $\mathrm{M}$ dwarf and late-K dwarf range in steps of $100 \mathrm{~K}$ were considered. All cases had $\log g=5$, except for the esd which had $\log g=5.5$. The visible and far-red portions of the spectra are plotted and compared in Fig. 3. The bands used to compute the $\mathrm{CaH}$, $\mathrm{TiO} 5$, and $\mathrm{CaOH}$ indices are indicated. The current BTSETTL line lists do not actually include $\mathrm{CaOH}$, but the bandpass also includes the $\mathrm{TiO} \gamma$ line which is also sensitive to $\mathrm{C} / \mathrm{O}$.

As expected, in the $\mathrm{C} / \mathrm{O}=1$ case (black lines in Fig. 3) the $\mathrm{TiO}$ bands at around 6235 and $6700 \AA$ are dramatically weakened relative to the solar case (red line) while those of $\mathrm{CaH}$ are essentially unchanged. This is also true for the esd case (but not the subdwarf case). The differential response of $\mathrm{TiO} 5$ and $\mathrm{CaH}$ is the basis for the " $\zeta$ " parameter developed by Lépine et al. (2007). However, the $\mathrm{C} / \mathrm{O}=1$ and esd cases differ markedly at $4700-5300 \AA$ and below $4500 \AA$ because Fe lines are very weak in the esd case. There are also difference at $\lambda>8000 \AA$ (Fig. 3). Absorption by $\mathrm{H}_{2} \mathrm{O}$ over a broad wavelength range centered at $1.9 \mu \mathrm{m}$ is also weaker in the $\mathrm{C} / \mathrm{O}=1$ case, but this would be difficult to ascertain from the ground. Index values were calculated for the four cases at each $T_{\text {eff }}$.

I searched for carbon-rich $\mathrm{M}$ dwarfs in three spectroscopic catalogs: the CONCH-SHELL spectroscopic catalog of nearby, bright $\mathrm{M}$ dwarfs (Gaidos et al. 2014), the Sloan Digital Sky Survey (SDSS) catalog of M dwarfs constructed by West et al. (2011), and the LAMOST spectroscopic catalog of M dwarfs in Yi et al. (2014). Stars in the CONCH-SHELL catalog were selected based on brightness $(J<9)$, parallaxes or proper motions con-

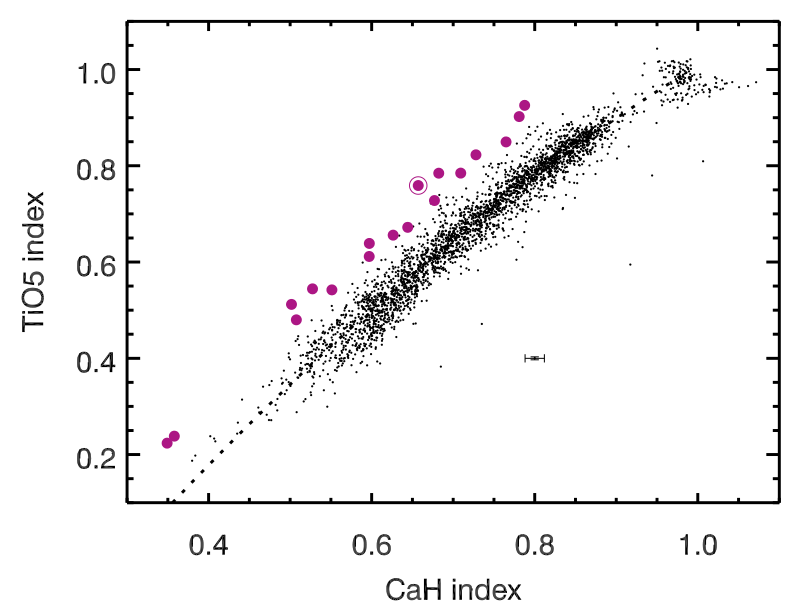

Figure 4. CaH vs. TiO5 indices for 2583 stars from the CONCHSHELL catalog of Gaidos et al. (2014), some with repeated observations. The black dashed curve is a quadratic fit to the locus. Eighteen stars with anomalously weak TiO5 bands (large indices) for their $\mathrm{CaH}$ band strength are marked as magenta points. The circled point (Wolf 1130 or GJ 781) has the most deviatory TiO5 index. The isolated point illustrates the median errors.

sistent with main sequence status, and colors. Although some sets of stars in the catalog were selected based on optical and infrared colors consistent with "normal" M dwarfs, this criterion was relaxed for another set, thus admitting any C-rich dwarfs with peculiar colors. The selection for "red" $(V-J>2.7)$ stars is not relaxed, and this inevitably eliminates some metal-poor early M-type dwarfs, although not necessarily C-rich examples. Figure 4 plots the TiO5 vs. CaH indices for CONCHSHELL stars. The red line is the best-fit quadratic to the locus and the black points and lines are the values computed from PHOENIX BT-SETTL spectra.

I identified 18 CONCH-SHELL stars with a TiO5 band that is significantly $(>3 \sigma)$ weaker (larger index) compared to a best-fit second-order fit of $\mathrm{TiO} 5$ as a function of $\mathrm{CaH}$. When calculating the significance of a deviation, an intrinsic dispersion of 0.032 in $\mathrm{TiO} 5$ was added in quadrature to the formal measurement errors. None of these 18 stars had indices that deviate to the extent predicted for a $\mathrm{C} / \mathrm{O}=1$ star. The most deviant star is PM I20050+5426/GJ 781/Wolf 1130, with a TiO5 index that is 0.17 above the best-fit locus value. This was previously identified as an active M1.5 subdwarf (Gizis 1997) with a metallicity based on an infrared spectrum of $-0.64 \pm 0.17$ (Roias-Avala et al. 2012). Interestingly, Gizis (1998) identified Wolf 1130 as a single-lined spectroscopic binary $(P \approx 0.5 \mathrm{~d})$ and proposed that the unseen companion was a $0.3 \mathrm{M}_{\odot}$ helium white dwarf. A Hubble Space Telescope Imaging Spectrograph (STIS) spectrum of the star (Fig. 5) exhibits some TiO absorption and no diagnostic carbon-star features. There is also no indication of a WD companion, although this would be consistent with an advanced age and hence low UV luminosity of any such object. Spectra of the other candidates indicate they are metal-poor stars, or have systematic errors.

I examined the molecular indices for two much larger samples of $\mathrm{M}$ dwarfs from the SDSS and LAMOST 


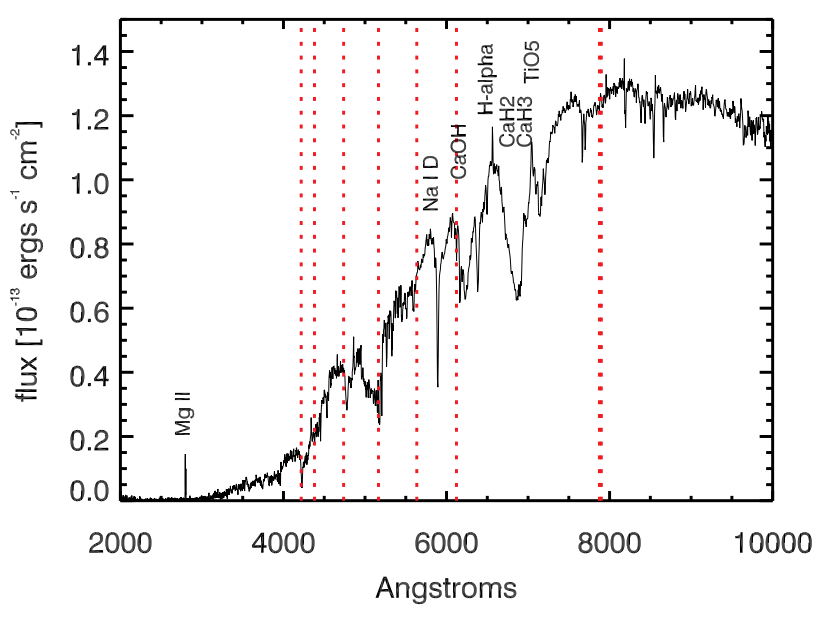

Figure 5. HST STIS spectrum of Wolf 1130/GJ 781, the star with the comparatively weakest TiO5 band in the catalog (circled point in Fig. 4). Some atomic lines and molecular bands are labeled; the red dashed lines mark the locations of some $\mathrm{CN}$ and $\mathrm{C}_{2}$ Schwan bands observed in carbon stars, but not in this star.

(West et al. 2011; Yi et al. 2014). Figure 6] shows the TiO5 and $\mathrm{CaOH}$ indices vs. $\mathrm{CaH}$ index for $70,841 \mathrm{M}$ dwarfs with spectra in Data Release 7 of the Sloan Digital Sky Survey (West et al. 2011). The grey scale is linearly related to the density of stars in the TiO5- or $\mathrm{CaOH}-\mathrm{vs}$. CaH plots. The black dashed line is the bestfit to the stellar locus, and the red, aquamarine, dashed purple, and black solid lines are the predictions from the BT-SETTL models for the solar metallicity, subdwarf, extreme subdwarf, and $\mathrm{C} / \mathrm{O}=1$ cases, respectively. Stars for which either index value is significantly $(>3 \sigma)$ higher than the best-fit polynomial to the stellar locus, and within $1 \sigma$ of or above the predicted $\mathrm{C} / \mathrm{O}=1$ locus and are plotted as open points. The 30 stars where both indices satisfy these criteria are plotted as filled points. Inspection of the SDSS spectra of these 30 stars found that all are consistent with template spectra of solar-metallicity or metal-poor stars and do not have the featuers expected of $\mathrm{C} / \mathrm{O}=1$ stellar atmospheres.

Figure 7 is the analogous set of plots for 67,082 candidate $\mathrm{M}$ dwarfs from a pilot survey of the Large Area Multi-Object Fiber Spectroscopic Telescope (LAMOST, Yi et al. 2014). The intrinsic scatter in index values, after correcting for measurement errors, is much larger for this sample, perhaps due to systematic errors. Only two stars have both $\mathrm{TiO} 5$ and $\mathrm{CaOH}$ values significantly above the locus, and within $1 \sigma$ of the predicted $\mathrm{C} / \mathrm{O}$ line. However, the index values of these stars are all $\gg 1$ and probably spurious. The paucity of high $\mathrm{C} / \mathrm{O}$ candidates in the LAMOST survey compared to the SDSS DR7 sample is undoubtedly due to the larger uncertainties in the indices.

Based on the CONCH-SHELL sample alone, high $\mathrm{C} / \mathrm{O}$ $\sim 1$ stars constitute less than $1.2 \times 10^{-3}$ of $\mathrm{M}$ dwarfs with $95 \%$ confidence, a stricter constraint than the limits placed by Nissen et al. (2014) based on spectroscopy of 66 solar-type stars. The null result from the DR7 sample places an an even more stringent upper limit of $6 \times 10^{-4}$ at $99 \%$ confidence. It is possible that manual screening performed by West et al. (2011) to remove corrupted

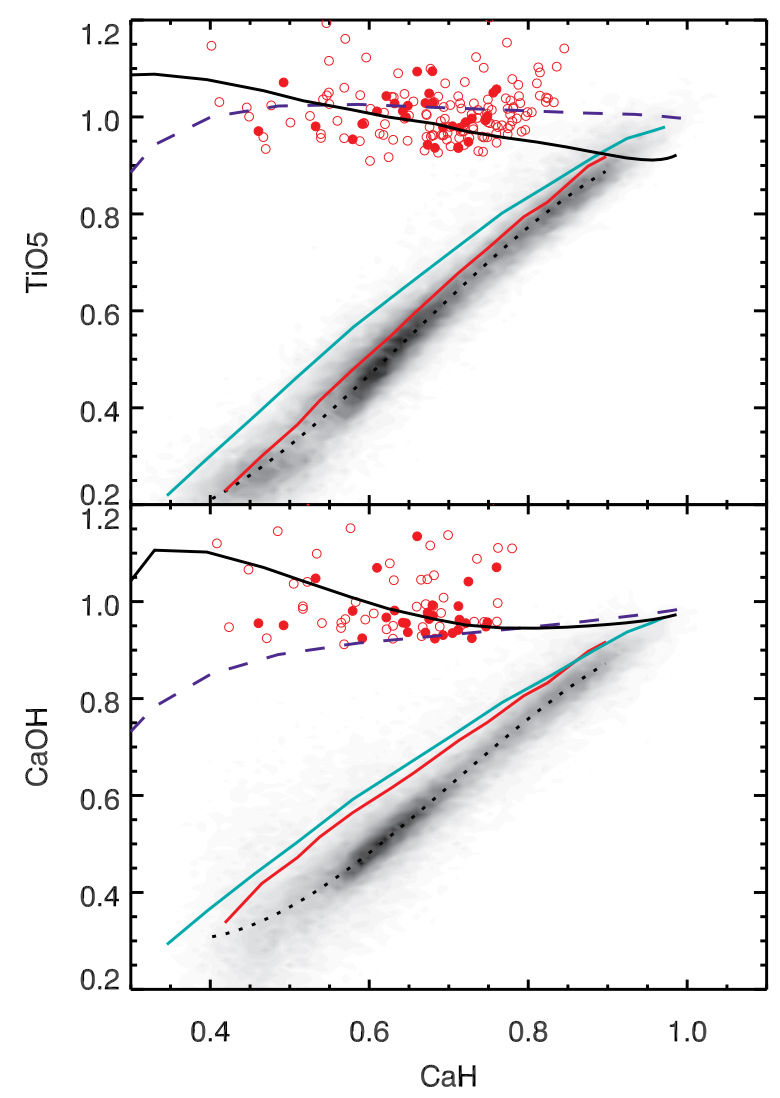

Figure 6. $\mathrm{CaH}, \mathrm{TiO} 5$, and $\mathrm{CaOH}$ indices of $\mathrm{M}$ dwarfs in Data Release 7 of the SDSS (West et al. 2011). The density of stars is shown as a grey scale. The dashed black curves are the best fits to the loci. The red, blue, purple dashed, and black solid curves are the predictions of the PHOENIX BT-SETTL model for solar-metallicity, subdwarf, extreme subdwarf, and $\mathrm{C} / \mathrm{O}=1$ cases, respectively.

spectra could have excluded carbon-rich stars, but at the resolution and signal-to-noise of the spectra, the differences that distinguish $\mathrm{C} / \mathrm{O}=1$ spectra from the solar case would not make them overwhelmingly abberant.

\section{FROM DUST TO PLANETESIMALS}

Theoretical studies linking the abundances in the photospheres of host stars to the composition of rocky planets generally assume that the building blocks of planetesimals condensed directly from a cooling gaseous disk of the same composition, but there is accumulating meteoritic evidence that primitive solids in the Solar System were by and large not produced by de nova condensation from a gas of solar composition. Instead, this material was the outcome of partial thermal processing of pre-existing solids under high dust/gas ratios and oxygen abundance (fugacity, $f_{\mathrm{O}_{2}}$ ) brought about by growth and settling of grains to the nebular mid-plane and/or inward migration of water-rich (and hence O-rich) planetesimals (Grossman et al. 2008). This evidence includes (i) the survival of pre-solar grains and isotopic anomalies inherited from the molecular cloud and older generations of stars (Davis 2011); (ii) the gradual variation in the abundance of elements with condensation temperature in primitive meteorites, which contradicts the sharp cutoff 


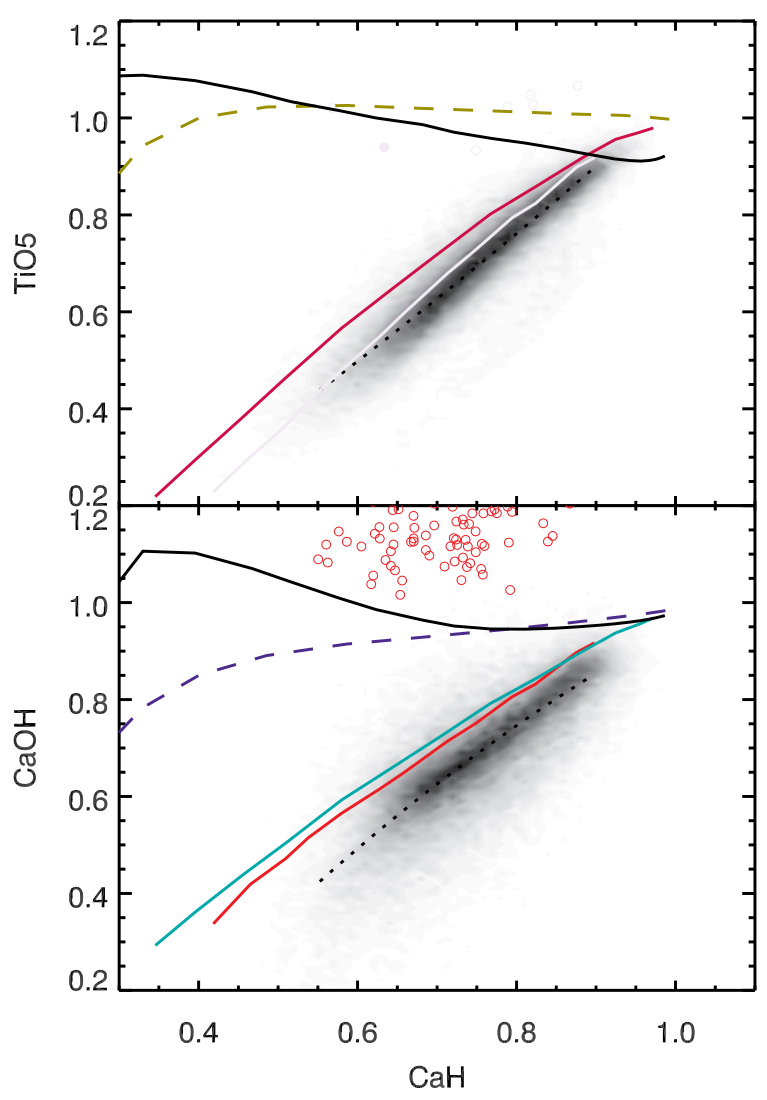

Figure 7. Same as Figure 6, but for candidate $M$ dwarfs from the LAMOST sample. Several stars with anomalous index values lie above the plots.

predicted by equilibrium condensation and which cannot be explained by radial transport in the disk (Ciesla 2008); (iii) the retention of volatile sodium and sulfur and lack of isotopic mass fractionation for potassium and silicon in chondrules (Scott 2007); and (iv) molybdenum and tungsten depletions in refractory inclusions (Fegley \& Palme 1985). The equilibrium oxygen fugacities of minerals in many meteorites also suggest high ambient $f_{\mathrm{O}_{2}}$, but some could also be explained by alteration on the parent bodies of the meteorites (Grossman et al. 2008).

Dust growth and settling to the midplane of a disk is predicted to occur in $10^{3}-10^{5} \mathrm{yr}$, depending on the intensity of turbulence in the disk (Nomura \& Nakagawa 2006; Ciesla 2007). Dust settling, as well as growth, might be observed via its effect on the spectral energy distribution of a disk (Tanaka et al. 2005). Tentative evidence for significant dust settling and depletion from the upper layers of the disks of $T$ Tauri stars (ages of $\sim 10^{6} \mathrm{yr}$ ) has been presented (Furlan et al. 2006), but unambiguous detection of settling is challenging (Murakawa 2014). If settling occurs faster than the viscous accretion time of a disk ( $10^{6} \mathrm{yr}$, Hartmann et al. 1998) then grains will experience high temperatures only in a dust-rich environment.

If the precursor material of planetesimals is dust, rather than gas, then to a large extent the composition of interstellar grains governs the composition of rocky exoplanets. Interstellar dust begins its existence as con- densates in the cooling envelopes and winds of AGB and red giant branch stars but these grains are subsequently and completely altered by many cycles of erosion and formation of mantles in the ISM. Erosion takes place in the lower-density, higher-temperature inter-cloud phase of the ISM, principally by sputtering by ions heated by the passage of supernova shocks as well as UV photons. Condensation takes place onto surviving grains that are incorporated into the denser, cooler cloud phase of the ISM. The cycling time between these two phases ( $\sim 3 \times 10^{7} \mathrm{yr}$, set by the cloud lifetime) is short compared to the mean time since formation in a circumstellar wind $\left(\sim 3 \times 10^{9} \mathrm{yr}\right)$, hence the bulk elemental composition of ISM grains is set by the balance between condensation and sputtering (Draine 2003).

The bulk composition of dust can be inferred by measuring the depletion of elements from the gas phase, i.e. using the strength of UV absorption lines along different lines of sight through the ISM to some suitable background source. Following Tielens (1998), the equations of motion of the depletions $\delta$ in the cloud $(c)$ and intercloud $(i)$ medium of any particular element can be written as:

$$
\frac{\delta_{c}}{d t}=-k_{2}\left(\delta_{c}-\delta_{i}\right)+k_{4}\left(1-\delta_{c}\right),
$$

and

$$
\frac{\delta_{i}}{d t}=-k_{1}\left(\delta_{i}-\delta_{c}\right)-k_{3} \delta_{i}
$$

respectively. In these equations, $k_{1}, k_{2}, k_{3}$, and $k_{4}$ are the rate of mixing from the intercloud to the cloud medium, the rate of mixing from the cloud to the intercloud medium, the rate of grain destruction in the intercloud medium, and the rate of grain growth from molecular cloud gas, respectively. The nucleosynthetic rate of production of an element is much slower than any of the rates of the formation of clouds, dissipation of clouds, destruction by sputtering in the inter-cloud phase, and growth condensation in the cloud phase, respectively, and is ignored here. The steady state solutions are:

$$
\delta_{c}=\frac{1+k_{1} / k_{3}}{1+k_{1} / k_{3}+k_{2} / k_{4}},
$$

and

$$
\delta_{i}=\frac{k_{1} / k_{3}}{1+k_{1} / k_{3}+k_{2} / k_{4}} .
$$

Thus, the steady-state abundances are governed by only two parameters, the amount of growth in the clouds $k_{4} / k_{2}$, and the amount of erosion between clouds: $k_{3} / k_{1}$.

I estimated parameter values for some elements using the data compiled by Jenkins (2009). I set $\delta_{c}$ and $\delta_{i}$ to $1-[X / H]_{1}$ (maximum depletion) and $1-[X / H]_{0}$ (minimum depletion), respectively, and solved for $k_{3} / k_{1}$ and $k_{4} / k_{2}$. Figure 8 plots these two parameters. Three elements with data in Jenkins (2009) are not shown: to explain the abundances of $\mathrm{P}, \mathrm{Cl}$, and $\mathrm{Zn}$ in the context of this model requires negative destruction in the intercloud medium. This may be an artifact of photoionization since the abundance of these elements is estimated from their singly ionized forms.

The comparatively low condensation rates of $\mathrm{Kr}, \mathrm{C}$, and $\mathrm{O}$ reflect the volatility of these elements. The behavior of $\mathrm{O}$ deviates strongly from that of the refractory 
elements, perhaps because the primary carrier of $\mathrm{O}$ is not silicates but a much more volatile substance such as water ice. Likewise $\mathrm{C}$ does not behave as a refractory such as graphite (Tielens 2012b). This suggests that $\mathrm{C}$ is present as relatively volatile organic matter (Jones 2009). $\mathrm{Kr}$ is not expected to condense and the non-zero value of $k_{4} / k_{2}$ may be a consequence of measurement errors or departures from solar relative abundances (Cartledge et al. 2003).

The differences in the parameters for $\mathrm{C}$ and $\mathrm{O}$ manifest themselves as a modified $\mathrm{C} / \mathrm{O}$ ratio in interstellar dust with respect to the total (gas+dust) abundance. In the case of the nominal rates inferred from the data of Jenkins (2009), the degree of depletion from cold molecular cloud gas shows that the $\mathrm{C} / \mathrm{O}$ of the dust is 0.92 times that of the bulk ISM, i.e. slightly more oxygen-rich than the current bulk ISM value. Although $\mathrm{O}$ is depleted more rapidly from interstellar grains, it is also accreted more rapidly. One caveat of this estimate for the dust $\mathrm{C} / \mathrm{O}$ is that the gas-phase depletion of $\mathrm{C}$ is more uncertain than other elements because there are few suitable absorption lines (Sofia \& Parvathi 2009).

The $\mathrm{C} / \mathrm{O}$ could also vary between locations as a result of varying $k_{3} / k_{1}$ and/or $k_{4} / k_{2}$. Tielens (1998) estimated the residence time $1 / k_{2}$ in the warm inter-cloud phase of the ISM as $3 \times 10^{6} \mathrm{yr}$ assuming that it is set by the timescale for shocking by SN and subsequent cooling and collapse into clouds. This was consistent with an ISM mass fraction in the warm phase of $\sim 10 \%$. However, the mass in the warm phase is probably comparable to the dense molecular $\mathrm{H}_{2}$ (cloud) phase Draine (2011) and thus the residence time in the inter-cloud phase is similar to the cloud lifetime $3 \times 10^{7}$ yr (Murray 2011). Over this time, dust grains may experience $\sim 10 \mathrm{SN}$ shocks before becoming incorporated into molecular clouds. In contrast, $k_{4} / k_{2}$ would be expected to vary only to the extent that the lifetimes of molecular clouds varies.

I estimated the sensitivity to variations in these rates by multiplying each of the parameter ratios by varying factors $\in[0,3]$. Figure 9 plots the predicted variation of $\mathrm{C} / \mathrm{O}$ with contours intervals of $10 \%$. The unadjusted parameter values, which predict $[\mathrm{C} / \mathrm{O}]_{\text {dust }}=0.92$ (heavy contour), are at unit value abcissa and ordinate (circle). Variation in the efficiency of grain destruction in the intercloud medium $\left(k_{3} / k_{1}\right)$ have more effect on dust $\mathrm{C} / \mathrm{O}$ than variation in the efficiency of grain growth in molecular clouds $\left(k_{4} / k_{2}\right)$. This difference is a consequence of the larger dispersion (a ratio of $\sim 30$ ) in the removal of $\mathrm{O}$ vs. C during grain destruction compared to the dispersion in the incorporation of $\mathrm{O}$ vs. $\mathrm{C}$ during grain growth (a ratio of $\sim 3$, Fig. 8). The very volatile behavior of $\mathrm{O}$ compared to the other elements is presumably because some of it is incorporated as water ice mantles around dust grains.

This model predicts that large (factors of two) variation in dust lifetime produced by different shock and UV conditions in the intercloud medium will produce modest $(\sim 20 \%)$ variation in bulk dust $\mathrm{C} / \mathrm{O}$, with dust in the vicinity of massive SN progenitors more carbon-rich. By the arguments presented above, variation in dust $\mathrm{C} / \mathrm{O}$ could generate diversity in the composition of planeteismals, but this will be limited by the extent that water ice is retained on dust grains (see below).

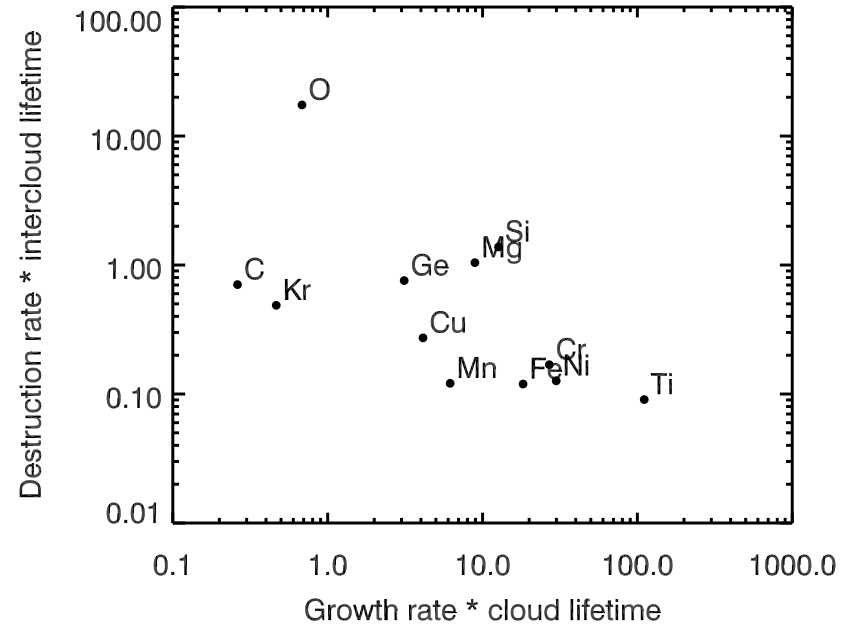

Figure 8. Inferred rates of growth (in molecular clouds) and depletion (in the inter-cloud medium) of elements in interstellar grains based on the observations analyzed in Jenkins (2009). The rates are normalized by the residence time of grains in the cloud and inter-cloud media, respectively.

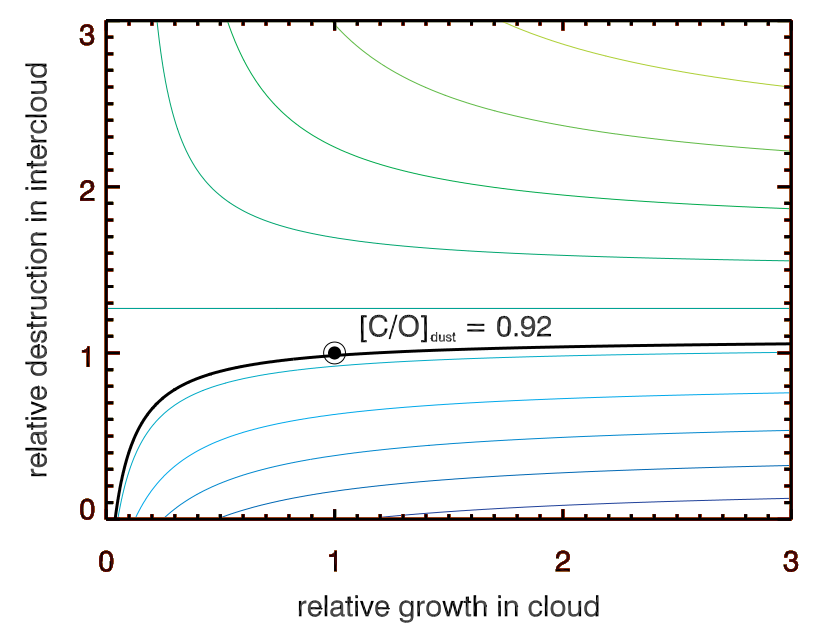

Figure 9. Predicted variation of $\mathrm{C} / \mathrm{O}$ in interstellar dust with rates of dust accretion and destruction relative to canonical parameter values, using the model of Tielens (1998) and canonical values set by the observed depletions in the ISM. Contours are intervals of 0.1 , increasing upwards, and the canonical rates yield a $\mathrm{C} / \mathrm{O}$ of 0.92 . Rates for each element are assumed to vary by a uniform multiplicative factor.

\section{ELEMENTAL ABUNDANCE VARIATION AMONG (PLANET-HOSTING) STARS}

A pronounced correlation between the metallicity of the host star and the occurrence of giant planets was discovered early in the history of exoplanet science (Gonzalez 1998). Increasingly precise measurements of the relative abundances of elements in stellar photospheres have permitted more nuanced investigations of correlations with planet occurrence (e.g., Robinson et al. 2006). Meléndez et al. (2009) found that the solar composition was anomalous compared to 9 out of a sample of 11 solar "twins" and that the Sun is deficient $(\leq 0.1 \mathrm{dex})$ in refractory elements (condensation temperature $\left.T_{C}>900 \mathrm{~K}\right)$ compared to more volatile elements. 
They proposed that this was a signature of the sequestration of those elements in rocky planets and cores of gas giants. Ramírez et al. (2010) also found a statistical correlation between planets and a deficit of refractory elements among larger samples of solar-type stars in Doppler surveys. If differences in relative photosphere abundances are a signature of rocky planets or giant planet cores, this would provide a short-cut to planet detection and even a means to probe the composition of the planets themselves (Delgado Mena et al. 2010).

On the other hand, González Hernández et al. (2013) find no clear correlation between the slope of the relative abundance vs. $T_{C}$ and the presence or absence of planets. One factor that may contribute to these contrasting results is the removal of galactic chemical evolution trends, e.g. decreasing $[\alpha / \mathrm{Fe}\rceil$ with increasing metallicity, by González Hernández et al. (2013). Moroever, Onehag et al. (2014) found no trend with $T_{C}$ in the relative abundances of members of the solar-age, solar-metallicity cluster M67. Observations of physical pairs of stars with and without (detected) planets provide the clearest test of the planet-abundance relation, as both stars should have formed from the same molecular cloud. But the available observations do unambiguously support such a correlation (Tucci Maia et al. 2014; Liu et al. 2014).

In fact, the trend of increasing differential abundance with increasing condensation temperature approximately mirrors the pattern of depletion of elements in ISM gas (Yin 2005). This simply reflects the preferential condensation of refractory elements onto interstellar grains and the universality of chemistry. If interstellar dust does not perfectly follow the gas, this will produce variation in element abundances that are proportional to the extent of gas-dust segregation and the dust composition. All else being equal, the abundances of the more refractory elements (which are mostly in the dust) would be expected to vary more than volatile elements (which are mostly in the gas), in a manner that roughly correlates with $T_{C}$.

There is evidence for, and theoretical predictions of, dust-gas segregation in the ISM. Padoan et al. (2006) found that the power-law describing the spatial distribution of extinction (a tracer of the column density of dust) on the sky is shallower than that of ${ }^{13} \mathrm{CO}$ (a tracer of the gas column density), such that in the densest regions of the ISM, extinction by dust is less than that predicted by perfect correspondence with the gas. This could be explained by grain growth in excess of that predicted by models. (CO condensation would presumably act in the opposite sense Whittet et al. 2010). But Padoan et al. (2006) offer spatial variation in dust-to-gas ratio as an alternative explanation.

Dust-gas segregation could occur at three different scales; (i) over the extent of a giant molecular cloud or star-forming region; (ii) in cloud cores that collapse to form individual stellar systems; and (iii) in the accretion disks around young stellar objects. Draine (2011) estimated that dust drift due to radiation pressure from $\mathrm{O}$ stars can remove dust from the centers of $\mathrm{H}$ II regions in $\leq 1 \mathrm{Myr}$, but since such low-density regions are not themselves the site of star formation, this effect should not manifest itself in the relative abundances of stellar photospheres.
Bellan (2008) showed that the different dynamics of dust and gas during Bondi-type accretion flow can enhance dust-to-gas ratios by an order of magnitude in cloud cores. The mechanism modeled by Bellan (2008) relies on dust velocities of several $\mathrm{km} \mathrm{sec}^{-1}$ relative to the gas around the growing cloud core. The size distribution of interstellar dust grains peaks sharply near 0.2 $0.3 \mu \mathrm{m}$ (Draine 2003). These grains may be dynamically decoupled from the diffuse interstellar medium (number density $n \sim 0.1 \mathrm{~cm}^{-3}$, stopping distance $\sim 40 \mathrm{pc}$ ), and there is tentative evidence for this in the trajectories of interstellar meteors (Tavlor et al. 1996) and Ulysses spacecraft measurements (Krüger \& Grün 2009). However, these grains will be tightly coupled to the molecular gas $\left(n \sim 10^{2}-10^{3} \mathrm{~cm}^{-3}\right)$ surrounding a cloud core, i.e. over length scales $\ll 1 \mathrm{pc}$ and much smaller than a typical cloud size. Under such conditions it seems unlikely that dust acceleration mechanisms (Yan 2009; Hoang et al. 2012, e.g.,) can achieve equipartion of energy between ISM and dust and speeds of $\sim 10 \mathrm{~km} \mathrm{~s}^{-1}$.

The second scenario which could produce variation in the dust-to-gas ratio in cloud cores is drift induced by radiation pressure. Whitworth \& Bate (2002) showed that the inward radial drift of $0.1-\mu \mathrm{m}$ grains under the influence of a typical radiation field can treble the dust concentration in a static gas sphere of a few solar masses at $10 \mathrm{~K}$ in 10 Myr. The effect scales linearly with the intensity of the external radiation field and the inverse of the characteristic cloud column density. Grains reach terminal velocity on a timescale of $10^{2} \mathrm{yr}$, i.e. much shorter than the cloud lifetime. Thus any density enhancement can be expected to grow linearly and the total dust enhancement can be expected to scale with the total external radiation experienced by the cloud core over its lifetime. Seo et al. (2011) numerically simulated this effect, including gas dynamics as well as coupling between the gas and dust, and found that the dust concentration is enhanced by about an order of magnitude in a narrow, inward-propagating shell, and depleted exterior to that shell. While the mean dust-to-gas ratio of a cloud is not changed by migration, truncation of the cloud by photoevaporation or internal collapse (to the exclusion of outer regions) would produce a metal-enhanced object. Both Whitworth \& Bate (2002) and Seo et al. (2011) point out the relevance of this process to the metallicities of stars and the formation of their planets.

A third scenario for dust-gas segregation involves accreting protostars and their disks, or concomitant Bondi-Hoyle accretion from the molecular cloud (Throop \& Bally 2008). Photoevaporation of gas, but not dust, from a disk produces an enhancement in the dust-to-gas ratio which is inherited by the star as disk accretion continues. Photoevaporation of disks driven by X-rays from the central star has been proposed to explain the final, rapid stage of circumstellar disk clearing (Owen et al. 2012). Observations of blue-shifted lines of Ne II (Pascucci \& Sterzik 2009) and O I (Hartigan et al. 1995) indicate that heavy volatile elements as well as $\mathrm{H}$ and $\mathrm{He}$ are lost in these winds. The temperature of the X-ray-heated "surface" of the disk from which the winds flow is heated to a few thousand K (Owen et al. 2012), but gravitational settling (and the formation of planetesimals) keeps dust near the cooler mid-plane. Disk masses 
are typically $1 \%$ of the central star, with a large scatter (Andrews et al. 2013); assuming the convection zone of a young solar-mass star contains $0.1 \mathrm{M}_{\odot}$, accretion of an entire disk which has been severaly gas-depleted would increase relative elemental abundances by $<0.04 \mathrm{dex}$, or $<0.01$ dex if the convection zone contains $0.4 \mathrm{M}_{\odot}$. This may fall short of explaining some of the observations.

Two important clues to the mechanism(s) of the observed variation are the spatial scale on which the variation occurs, and the $T_{C}$ of the "elbow" in the relative abundance variation below which abundances do not vary in any systematic manner. Surveys of nearby, solar-age field stars offer little information on the spatial scale of the segregation as the stars are far from their birthplace. Onehag et al. (2014) found no significant difference between M67 cluster stars and the Sun, and proposed that the Sun also formed in a dense cluster analogous to M67. They suggested that the common pattern of relative abundances was established by the removal of dust by radiation drift. But there are several problems with this explanation. Star formation does not take place in HII regions but in surrounding neutral gas, and, as they point out, the expansion of an HII region should outpace dust drift. This explanation also requires most stars to form after an earlier generation of massive stars that move the dust. It also requires that the vast majority of nearby solar-type stars have not formed in dense stellar clusters, something not supported by cluster statistics (Williams \& Gaidos 2007). Finally, abundance differences between stars in physical pairs requires gasdust segregation on scales smaller than the cloud core.

The other clue is the value $T_{C}$ below which relative abundance variation disappears, as this suggests the temperature and hence location where the dust and gas segregation occurs. If this "knee" in the abundance variation vs. $T_{C}$ is $1000 \mathrm{~K}$, as many data sets suggest (Meléndez et al. 2009), this would seem to rule out a molecular cloud setting with $T \sim 20-100 \mathrm{~K}$ where even some $\mathrm{C}$ and $\mathrm{O}$ condense as organics and ices. All of these observations point to gas-dust segregation during the formation or subsequent evolution of an accretion disk as a plausible explanation of the abundance trends.

\section{DISCUSSION}

Neither observations of M dwarfs nor models of Galactic chemical evolution support the premise that there are a significant number of stellar systems with primordial $\mathrm{C} / \mathrm{O} \sim 1$ in the solar neighborhood, i.e. within the samples of Doppler radial velocity surveys. Moreover, if the process of planet formation is universal, then studies of primitive meteorites show that the chemical composition of small, rocky planets is controlled largely by the composition and thermal processing of pre-existing dust from the parent molecular cloud, rather than condensation from gas with the stellar composition, and that this dust is likely to have a $\mathrm{C} / \mathrm{O}$ reflecting that of the bulk ISM. Finally, small differences in the relative patterns between the Sun and solar "twins" can best be explained by dust-gas segregation at stellar scales and temperatures of up to $1000 \mathrm{~K}$, i.e. in accreting protostars, rather than planet formation per se.

When comparing the Sun to the solar neighborhood it is usually assumed that they share a common history. However, it is also possible that the two are not related. Nieva \& Przybilla (2012) estimated "cosmic" standard abundances (rather the present-day abundances in the solar neighborhood) using bright, slowlyrotating, early-type B stars. Due to their short main sequence lives, B stars contain abundance information that is both contemparaneous and local, and their purely radiative atmospheres are comparatively simple to model, although UV photoionization rates must be correctly modeled (Lyubimkov 2013). Nieva \& Przybilla (2012) report metallicities that are very close to solar, depsite 4.5 Gyr of intervening GCE, and a mean $\mathrm{C} / \mathrm{O}$ of 0.37 , lower than the solar value of 0.55 . They explain both discrepancies by appealing to outward migration of the Sun in the galactic disk. These findings warrant further investigation.

Depletion patterns in the ISM suggest that the $\mathrm{C} / \mathrm{O}$ of dust largely reflects the ISM; $\mathrm{O}$ is incorporated more rapidly in grains than $\mathrm{C}$ in molecular clouds but it is depleted from grains more quickly in the intercloud medium, and these two effects approximately balance. Variation in the efficiency of grain erosion by SN shocks and $\mathrm{UV}$ radiation could produce modest variations in the $\mathrm{C} / \mathrm{O}$, however. While the phases of $\mathrm{O}$ in interstellar grains are clearly water ice and silicates, the phases of $\mathrm{C}$ are more controversial. Volatility patterns suggest $\mathrm{C}$ is not in refractory phase(s) like graphite and carbides but instead more volatile organic molecules, in particular aromatic hydrocarbons (Jones 2009; Jones et al. 2013; Chiar et al. 2013). Aliphatic hydrocarbons have been suggested as the source of the ubiquitious ISM absorbtion feature at $3.4 \mu \mathrm{m}$ but the absence of polarization in this line is difficult to reconcile with a scenario of condensation of carbonaceous mantles onto silicate cores ( $\mathrm{Li}$ et al. 2014). Surface reactions could explain why growth rate of $\mathrm{C}$ in grains is very slow with respect to other elements.

While these considerations plus a bulk ISM $\mathrm{C} / \mathrm{O}<1$ are sufficient to explain the oxidized nature of dust in the Solar System, it remains to be explained why primitive meteorites in the Solar System are very depleted in both $\mathrm{C}$ and $\mathrm{O}$, especially $\mathrm{C}$. Based on the meteorite abundances and associated uncertainties compiled by Lodders (2003) and solar abundances and uncertainties from Caffau et al. (2011), the depletion has a stochiometric ratio of $0.93 \pm 0.34$, i.e. consistent with unity and depletion by the formation and removal of $\mathrm{CO}$. In this scenario the removal of $\mathrm{C}$ or $\mathrm{O}$ is not controlled by volatility, otherwise either $\mathrm{C}$ or $\mathrm{O}$ would be far more depleted than the other, depending on the effective $T_{C}$. This can occur if the reaction occurs in situ, i.e. at temperatures lower than the $T_{C}$ s of the $\mathrm{C}$ and $\mathrm{O}$ phases $(\sim 100 \mathrm{~K})$ where both elements are retained in grains, and/or where vapor is not lost by turbulent mixing, i.e. in "dead zone" where turbulence is suppressed.

Primitive meteorites, considered analogs to the nowlost building blocks of the Solar System, also include the highly-reduced enstatite chondrites. Enstatite chondrites contain carbide minerals but virtually no water and have been explained by equilibration of solids with a gas having $\mathrm{C} / \mathrm{O}=0.83$ (Grossman et al. 2008). Interestingly, removal of an amount of $\mathrm{C}$ and $\mathrm{O}$ equal to that incorporated in CI chondrites produces a residual gas with a $\mathrm{C} / \mathrm{O}$ of 0.93 . Hutson \& Ruzicka (2000) have proposed that removal of refractories from a gas of solar composition and equilibration of solids with the remain- 
ing gas could produce the precursors for enstatite chondrites. If enstatite chondrites formed from recycling of the precursors of terrestrial planets in an oxygen-poor gas it could explain why the enstatite chondrites and Earth lie along the same mass-dependent fractionation line in a three oxygen-isotope plot. This equilibration would necessarily have occurred at a comparatively low dust-to-gas ratio and lower temperatures where moderately volatile elements such as Fe would not be lost.

$\mathrm{Al}-\mathrm{Mg}$ isotopes of CAIs in unequilibrated enstatite chondrites indicate that these refractory inclusions formed in the same region as CAIs in other chondrites, but were subsequently exposed to reducing conditions (Guan et al. 2000). Reducing conditions could have been established sequentially or in a different part of the protoplanetary disk. The formation times of enstatite chondrites is an area of active research. Dating of sulfides in unequilibrated $\mathrm{E}$ chondrites using the $\mathrm{Fe}-\mathrm{Ni}$ and $\mathrm{Mn}-\mathrm{Cr}$ short-lived radionuclide chronometers gives ages of 1213 Myr after CAI formation (Wadhwa et al. 1997). Reanalysis of the $\mathrm{Mn}-\mathrm{Cr}$ isotope data for a single sulfide in the MacAlpine Hills 88136 EL3 chondrite (Guan et al. 2007; Telus et al. 2012) plus an initial ${ }^{53} \mathrm{Mn} /{ }^{55} \mathrm{Mn}$ of $5.1 \times 10^{-6}$ (Yin et al. 2007) gives an age of $10 \mathrm{Myr}$.

In summary, I propose the following scenario for the chemistry of rocky planets:

- Planet formation proceeds from a two-component ISM (gas and dust) which are never fully equilibraxted during the formation process.

- The composition of the precursor material of Solar System material is interstellar dust with refractory abundances and a $\mathrm{C} / \mathrm{O}$ ratio approximately equal to the bulk ISM; this was set by grain growth in molecular clouds and destruction between clouds.

- Planetesimals, as represented by carbonaceous (CI) chondrites, formed under the oxidizing, high dustto-gas ratio conditions established by settling of interstellar grains to the disk mid-plane and subsequent depletion of $\mathrm{C}$ and $\mathrm{O}$ by formation and removal of CO.

- Enstatite chondrites represent reduced material that equilibrated with gas after removal of solids in approximately CI proportions, possibly during a second generation of planetesimal formation.

- The chemical composition of rocky exoplanets could be set by mixing of oxidized and reduced generations of planetesimals. The mixing ratio could be determined by the effiency with which the first, oxidizing generation incorporated disk solids, as well as the dynamics of accretion in the disk.

Giant planets, unlike the small, rocky planets considered in this work, accrete massive gas envelopes that would include volatile species such as $\mathrm{CO}$, and the considerations described above do not preclude the possibility of C-rich atmospheres in giant planets. Detections of such objects have been claimed, but are controversial (e.g. Madhusudhan et al. 2011; Crossfield et al. 2012; Swain et al. 2013; Line et al. 2014; Stevenson et al.
2014; Hansen et al. 2014). If the core-first model of giant planet formation is correct, removal of $\mathrm{O}$ as silicates and sequestration into a core increases the $\mathrm{C} / \mathrm{O}$ ratio of the gas that is subsequently captured into the planet's envelope. Beyond the ice-line, condensation of water and removal of more $\mathrm{O}$ would drive the $\mathrm{C} / \mathrm{O}$ of the gas even closer to unity (Oberg et al. 2011). Dissociation of CO in the disk and removal of the $\mathrm{O}$ as water ice would further enhance this ratio, and indeed the enrichment of planetary water in ${ }^{17} \mathrm{O}$ and ${ }^{18} \mathrm{O}$ relative to the Sun is thought to be a signature of this process (Clayton 2002). These processes may facilitate the formation of reduced, enstatite chondrite-like planetesimals as discussed above.

The author thanks Derek Homeier and France Allard for generating the $\mathrm{C} / \mathrm{O}=1$ cases with the PHOENIX atmosphere model, Gary Huss for discussions which stimulated this work, and Larry Nittler for comments. This research was supported by NASA grants NNX10AQ36G and NNX11AC33G to EG.

\section{REFERENCES}

Alexander, C. M. O., \& Ebel, D. S. 2012, Meteoritics and Planetary Science, 47, 1157

Allard, F., Homeier, D., \& Freytag, B. 2011, in Astronomical Society of the Pacific Conference Series, Vol. 448, 16th Cambridge Workshop on Cool Stars, Stellar Systems, and the Sun, ed. C. Johns-Krull, M. K. Browning, \& A. A. West, 91

Andrews, S. M., Rosenfeld, K. A., Kraus, A. L., \& Wilner, D. J. 2013, ApJ, 771, 129

Barshay, S. S., \& Lewis, J. S. 1976, ARA\&A, 14, 81

Beers, T. C., Preston, G. W., \& Shectman, S. A. 1992, AJ, 103, 1987

Behara, N. T., Bonifacio, P., Ludwig, H.-G., et al. 2010, A\&A, 513, A72

Bellan, P. M. 2008, ApJ, 678, 1099

Bensby, T., \& Feltzing, S. 2008, in Nuclei in the Cosmos (NIC X), 197

Bolatto, A. D., Wolfire, M., \& Leroy, A. K. 2013, ARA\&A, 51, 207

Bond, J. C., O'Brien, D. P., \& Lauretta, D. S. 2010, ApJ, 715, 1050

Burkhardt, C., Kleine, T., Dauphas, N., \& Wieler, R. 2012, Earth and Planetary Science Letters, 357, 298

Caffau, E., Ludwig, H.-G., Steffen, M., Freytag, B., \& Bonifacio, P. 2011, Sol. Phys., 268, 255

Carter-Bond, J. C., O'Brien, D. P., \& Raymond, S. N. 2012, ApJ, 760,44

Cartledge, S. I. B., Meyer, D. M., \& Lauroesch, J. T. 2003, ApJ, 597,408

Cescutti, G., Matteucci, F., McWilliam, A., \& Chiappini, C. 2009, A\&A, 505, 605

Chiar, J. E., Tielens, A. G. G. M., Adamson, A. J., \& Ricca, A. 2013, ApJ, 770, 78

Chomiuk, L., \& Povich, M. S. 2011, AJ, 142, 197

Ciesla, F. J. 2007, ApJ, 654, L159

Ciesla, F. J. 2008, Meteoritics \& Planetary Science, 43, 639

Clayton, R. N. 2002, Nature, 415, 860

Crossfield, I. J. M., Barman, T., Hansen, B. M. S., Tanaka, I., \& Kodama, T. 2012, ApJ, 760, 140

Dahn, C. C., Liebert, J., Kron, R. G., Spinrad, H., \& Hintzen, P. M. 1977, ApJ, 216, 757

Davis, A. M. 2011, Proceedings of the National Academy of Science, 108, 19142

Delgado Mena, E., Israelian, G., González Hernández, J. I., et al. 2010, ApJ, 725, 2349

Doherty, C. L., Gil-Pons, P., Lau, H. H. B., Lattanzio, J. C., \& Siess, L. 2014, MNRAS, 437, 195

Downes, R. A., Margon, B., Anderson, S. F., et al. 2004, AJ, 127, 2838 
Draine, B. T. 2003, ARA\&A, 41, 241

-. 2011, Physics of the Interstellar and Intergalactic Medium

Ecuvillon, A., Israelian, G., Santos, N. C., et al. 2004, A\&A, 426, 619

-. 2006, A\&A, 445, 633

Edvardsson, B., Andersen, J., Gustafsson, B., et al. 1993, A\&A, 275, 101

Elser, S., Meyer, M. R., \& Moore, B. 2012, Icarus, 221, 859

Esteban, C., García-Rojas, J., Carigi, L., et al. 2014, MNRAS, 443,624

Fabbian, D., Nissen, P. E., Asplund, M., Pettini, M., \& Akerman, C. 2009, A\&A, 500, 1143

Fegley, B., \& Palme, H. 1985, Earth and Planetary Science Letters, 72,311

Fischer, D. A., \& Valenti, J. 2005, ApJ, 622, 1102

Fortney, J. J. 2012, ApJ, 747, L27

Frayer, D. T., \& Brown, R. L. 1997, ApJS, 113, 221

Furlan, E., Hartmann, L., Calvet, N., et al. 2006, ApJS, 165, 568

Gaidos, E., Mann, A. W., Lepine, S., et al. 2014, ArXiv e-prints, arXiv:1406.7353

Gaidos, E. J. 2000, Icarus, 145, 637

Garnett, D. R., Skillman, E. D., Dufour, R. J., et al. 1995, ApJ, 443,64

Gehrz, R. D., Truran, J. W., Williams, R. E., \& Starrfield, S. 1998, PASP, 110, 3

Gizis, J. E. 1997, AJ, 113, 806

一. 1998, AJ, 115, 2053

Gonzalez, G. 1998, A\&A, 334, 221

González Hernández, J. I., Delgado-Mena, E., Sousa, S. G., et al. 2013, A\&A, 552, A6

Green, P. 2013, ApJ, 765, 12

Grossman, L., Beckett, J. R., Fedkin, A. V., Simon, S. B., \& Ciesla, F. J. 2008, Reviews in Mineralogy and Geochemistry, 68,93

Guan, Y., Huss, G. R., \& Leshin, L. A. 2007, Geochim. Cosmochim. Acta, 71, 4082

Guan, Y., Huss, G. R., MacPherson, G. J., \& Wasserburg, G. J. 2000, Science, 289, 1330

Gustafsson, B., Karlsson, T., Olsson, E., Edvardsson, B., \& Ryde, N. 1999, A\&A, 342, 426

Hansen, C. J., Schwartz, J. C., \& Cowan, N. B. 2014, MNRAS, 444, 3632

Hartigan, P., Edwards, S., \& Ghandour, L. 1995, ApJ, 452, 736

Hartmann, L., Calvet, N., Gullbring, E., \& D'Alessio, P. 1998, ApJ, 495, 385

Hezel, D. C., Russell, S. S., Ross, A. J., \& Kearsley, A. T. 2008, Meteoritics and Planetary Science, 43, 1879

Hoang, T., Lazarian, A., \& Schlickeiser, R. 2012, ApJ, 747, 54

Holmberg, J., Nordström, B., \& Andersen, J. 2007, A\&A, 475, 519

Huss, G. R., Meshik, A. P., Smith, J. B., \& Hohenberg, C. M. 2003, Geochim. Cosmochim. Acta, 67, 4823

Hutson, M., \& Ruzicka, A. 2000, Meteoritics and Planetary Science, 35, 601

Jao, W.-C., Henry, T. J., Beaulieu, T. D., \& Subasavage, J. P. 2008, AJ, 136, 840

Jenkins, E. B. 2009, ApJ, 700, 1299

Jones, A. P. 2009, in Astronomical Society of the Pacific Conference Series, Vol. 414, Cosmic Dust - Near and Far, ed. T. Henning, E. Grün, \& J. Steinacker, 473

Jones, A. P., Fanciullo, L., Köhler, M., et al. 2013, A\&A, 558, A62

Karakas, A. I. 2010, MNRAS, 403, 1413

Karakas, A. I., \& Lattanzio, J. C. 2014, ArXiv e-prints, arXiv: 1405.0062

Kobayashi, C., Karakas, A. I., \& Umeda, H. 2011, MNRAS, 414, 3231

Kobayashi, C., Umeda, H., Nomoto, K., Tominaga, N., \& Ohkubo, T. 2006, ApJ, 653, 1145

Krüger, H., \& Grün, E. 2009, Interstellar Dust Inside and Outside the Heliosphere, ed. J. L. Linsky, V. V. Izmodenov, E. Möbius, \& R. von Steiger, 347

Lépine, S., Rich, R. M., \& Shara, M. M. 2007, ApJ, 669, 1235

Li, Q., Liang, S. L., \& Li, A. 2014, MNRAS, 440, L56

Line, M. R., Knutson, H., Wolf, A. S., \& Yung, Y. L. 2014, ApJ, 783,70

Liu, F., Asplund, M., Ramírez, I., Yong, D., \& Meléndez, J. 2014, MNRAS, 442, L51

Lodders, K. 2003, Sciences-New York, 1220
Lyubimkov, L. S. 2013, Astrophysics, 56, 472

Madhusudhan, N., Harrington, J., Stevenson, K. B., et al. 2011 , Nature, 469, 64

Maeder, A. 1992, A\&A, 264, 105

Marcy, G. W., Isaacson, H., Howard, A. W., et al. 2014, ApJS, 210,20

Mattsson, L. 2010, A\&A, 515, A68

Meléndez, J., Asplund, M., Gustafsson, B., \& Yong, D. 2009, ApJ, 704, L66

Moriarty, J., Madhusudhan, N., \& Fischer, D. 2014, ApJ, 787, 81

Murakawa, K. 2014, Planet. Space Sci., 100, 51

Murray, N. 2011, ApJ, 729, 133

Naab, T., \& Ostriker, J. P. 2006, MNRAS, 366, 899

Nieva, M.-F., \& Przybilla, N. 2012, A\&A, 539, A143

Nissen, P. E. 2013, A\&A, 552, A73

Nissen, P. E., Chen, Y. Q., Carigi, L., Schuster, W. J., \& Zhao, G. 2014, ArXiv e-prints, arXiv:1406.5218

Nomura, H., \& Nakagawa, Y. 2006, The Astrophysical Journal, 640, 1099

Öberg, K. I., Murray-Clay, R., \& Bergin, E. A. 2011, ApJ, 743, L16

Önehag, A., Gustafsson, B., \& Korn, A. 2014, A\&A, 562, A102

Owen, J. E., Clarke, C. J., \& Ercolano, B. 2012, MNRAS, 422, 1880

Padoan, P., Cambrésy, L., Juvela, M., et al. 2006, ApJ, 649, 807

Pascucci, I., \& Sterzik, M. 2009, ApJ, 702, 724

Petigura, E. A., Howard, A. W., \& Marcy, G. W. 2013, Proceedings of the National Academy of Science, 110, 19273

Petigura, E. A., \& Marcy, G. W. 2011, ApJ, 735, 41

Portinari, L., Chiosi, C., \& Bressan, A. 1998, A\&A, 334, 505

Prantzos, N. 2008, Sciences-New York, 1

Rajpurohit, A. S., Reylé, C., Allard, F., et al. 2014, A\&A, 564 A90

Ramírez, I., Asplund, M., Baumann, P., Meléndez, J., \& Bensby, T. 2010, A\&A, 521, A33

Reid, I. N., Hawley, S. L., \& Gizis, J. E. 1995, AJ, 110, 1838

Renzini, A., \& Voli, M. 1981, A\&A, 94, 175

Robinson, S. E., Laughlin, G., Bodenheimer, P., \& Fischer, D. 2006, Astrophysical Journal, 643, 484

Rojas-Ayala, B., Covey, K. R., Muirhead, P. S., \& Lloyd, J. P. 2012, ApJ, 748, 93

Santos, N. C., Israelian, G., \& Mayor, M. 2004, A\&A, 415, 1153

Schmidt, S. J., Wallerstein, G., Woolf, V. M., \& Bean, J. L. 2009, PASP, 121, 1083

Scott, E. R. D. 2007, Annual Review of Earth and Planetary Sciences, 35, 577

Seo, Y. M., Kim, J., \& Hong, S. S. 2011, ApJ, 728, 140

Siess, L. 2010, A\&A, 512, A10

Silburt, A., Gaidos, E., \& Wu, Y. 2014, ArXiv e-prints, arXiv: 1406.6048

Sofia, U. J., \& Parvathi, V. S. 2009, in Astronomical Society of the Pacific Conference Series, Vol. 414, Cosmic Dust - Near and Far, ed. T. Henning, E. Grün, \& J. Steinacker, 236

Stevenson, K. B., Bean, J. L., Madhusudhan, N., \& Harrington, J. 2014, ApJ, 791, 36

Swain, M., Deroo, P., Tinetti, G., et al. 2013, Icarus, 225, 432

Tanaka, H., Himeno, Y., \& Ida, S. 2005, ApJ, 625, 414

Taylor, A. D., Baggaley, W. J., \& Steel, D. I. 1996, Nature, 380, 323

Telus, M., Huss, G. R., Ogliore, R. C., Nagashima, K., \& Tachibana, S. 2012, Meteoritics and Planetary Science, 47, 2013

Teske, J. K., Cunha, K., Schuler, S. C., Griffith, C. A., \& Smith, V. V. 2013a, ApJ, 778, 132

Teske, J. K., Cunha, K., Smith, V. V., Schuler, S. C., \& Griffith, C. A. 2014, ApJ, 788, 39

Teske, J. K., Schuler, S. C., Cunha, K., Smith, V. V., \& Griffith, C. A. 2013b, ApJ, 768, L12

Throop, H. B., \& Bally, J. 2008, AJ, 135, 2380

Tielens, A. G. G. M. 1998, ApJ, 499, 267

Tielens, A. G. G. M. 2012a, in IAU Symposium, Vol. 284, IAU Symposium, ed. R. J. Tuffs \& C. C. Popescu, 72-81

Tielens, A. G. G. M. 2012b, in IAU Symposium, Vol. 284, IAU Symposium, ed. R. J. Tuffs \& C. C. Popescu, 72-81

Timmes, F. X., Woosley, S. E., \& Weaver, T. A. 1995, ApJS, 98, 617

Tinsley, B. M. 1980, Fund. Cosmic Phys., 5, 287 
Trinquier, A., Elliott, T., Ulfbeck, D., et al. 2009, Science, 324, 374

Tucci Maia, M., Melendez, J., \& Ramirez, I. 2014, ArXiv e-prints, arXiv:1407.4132

Wadhwa, M., Zinner, E. K., \& Crozaz, G. 1997, Meteoritics and Planetary Science, 32, 281

West, A. A., Morgan, D. P., Bochanski, J. J., et al. 2011, AJ, 141, 97

Wheeler, J. C., Sneden, C., \& Truran, Jr., J. W. 1989, ARA\&A, 27,279

Whittet, D. C. B., Goldsmith, P. F., \& Pineda, J. L. 2010, ApJ, 720,259

Whitworth, A. P., \& Bate, M. R. 2002, MNRAS, 333, 679
Williams, J. P., \& Gaidos, E. 2007, The Astrophysical Journal, 663, L33

Wolfire, M. G., McKee, C. F., Hollenbach, D., \& Tielens,

A. G. G. M. 2003, ApJ, 587, 278

Woolf, V. M., Lépine, S., \& Wallerstein, G. 2009, PASP, 121, 117

Woosley, S. E., \& Weaver, T. A. 1995, ApJS, 101, 181

Yan, H. 2009, MNRAS, 397, 1093

Yi, Z., Luo, A., Song, Y., et al. 2014, AJ, 147, 33

Yin, Q. 2005, in Astronomical Society of the Pacific Conference Series, Vol. 341, Chondrites and the Protoplanetary Disk, ed. A. N. Krot, E. R. D. Scott, \& B. Reipurth, 632

Yin, Q.-z., Jacobsen, B., Moynier, F., \& Hutcheon, I. D. 2007, ApJ, 662, L43

\section{APPENDIX}

\section{GALACTIC CHEMICAL EVOLUTION MODEL}

I computed changes in the abundance of five isotopes in the vicinity of the solar galactocetnric radius: ${ }^{12} \mathrm{C}$, ${ }^{13} \mathrm{C}$, ${ }^{16} \mathrm{O},{ }^{17} \mathrm{O}$, and ${ }^{18} \mathrm{O}$. The model accounts for the production of these isotopes and their release into the ISM in SN explosions, winds from massive stars, and AGB winds, incorporation of isotopes into long-lived low-mass stars, and dilution of the ISM by the infall of metal-poor gas. The ISM is described by a two-box model with a lower-density, warmer intercloud medium (ICM) which spawns molecular clouds, and a giant molecular cloud (GMC) component that can form stars. The mean lifetime of molecular clouds is $\tau_{\text {GMC }}$ and the residence time of gas in the ICM before condensing into a cloud is $\tau_{\mathrm{ICM}}$. The equations of motion for the mass surface density $m$ of the two components are:

$$
\begin{gathered}
\frac{d m_{\mathrm{ICM}}}{d t}=-\frac{m_{\mathrm{ICM}}}{\tau_{\mathrm{ICM}}}+\frac{m_{\mathrm{GMC}}}{\tau_{\mathrm{GMC}}}+S_{\mathrm{ICM}}+S_{\mathrm{AGB}}+F, \\
\frac{d m_{\mathrm{GMC}}}{d t}=\frac{m_{\mathrm{ICM}}}{\tau_{\mathrm{ICM}}}-\frac{m_{\mathrm{GMC}}}{\tau_{\mathrm{GMC}}}+S_{\mathrm{GMC}}-R \mathcal{M},
\end{gathered}
$$

where $F$ is the infall rate, $R$ is the rate of formation rate of stars with $M>1 \mathrm{M}_{\odot}, \mathcal{M}$ is the ratio of the total stellar mass to the mass in stars $>1 \mathrm{M}_{\odot}, S_{\mathrm{ICM}}$ is the flux of SN ejecta into the ICM from progenitors that explode in time $T>\tau_{\mathrm{GMC}}, S_{\mathrm{GMC}}$ is the flux of SN ejecta into the parent molecular cloud from progenitors that contribute to GMCs in time $T<\tau_{\mathrm{GMC}}$, and $S_{\mathrm{AGB}}$ is the wind from AGB stars, all contributing to the ICM. The fluxes from AGB winds and SN ejecta are given by:

$$
S=\int_{M_{1}}^{M_{2}} \frac{R\left(T\left(M_{*}\right)\right)}{\langle M\rangle} E\left(M_{*}\right) f\left(M_{*}\right) d M_{*},
$$

where $\langle M\rangle$ is the mean mass of stars with $M_{*}>1 \mathrm{M}_{\odot}, E$ is the ejected mass in winds and/or explosions, $f$ is the fractional number of stars per unit mass in the IMF, and $M_{1}$ and $M_{2}$ are the minimum and maximum masses contributing mass to the three different cases, and $P$ is the mass in the wind or ejecta from a progenitor of mass $M_{*}$. The equations for the mass surface densities of stars $\left(m_{*}\right)$ and stellar remnants $\left(m_{r}\right)$ are

$$
\frac{d m_{*}}{d t}=R \mathcal{M}-\int_{M_{1}}^{M_{2}} R\left(T\left(M_{*}\right)\right) f\left(M_{*}\right) d M_{*},
$$

and

$$
\frac{d m_{r}}{d t}=\int_{M_{1}}^{M_{2}} \frac{R\left(T\left(M_{*}\right)\right)}{\langle M\rangle}\left[M_{*}-P\left(M_{*}\right)\right] f\left(M_{*}\right) d M_{*},
$$

where the limits of integation are over any stars that are moving off the main sequence. The star formation rate is related to the total mass surface density of gas using a Schmidt-Kennicut law:

$$
R=R_{0}\left(\frac{m_{\mathrm{GMC}}+m_{\mathrm{ICM}}}{m_{0}}\right)^{\beta}
$$

The equations governing the the mass fraction of the $i$ th isotope is:

$$
\begin{gathered}
m_{\mathrm{ICM}} \frac{d X_{\mathrm{ICM}}^{i}}{d t}=\frac{m_{\mathrm{GMC}} X_{G M C}^{i}}{\tau_{\mathrm{GMC}}}-\frac{m_{\mathrm{ICM}} X_{\mathrm{ICM}}^{i}}{\tau_{\mathrm{ICM}}}+S_{\mathrm{ICM}}^{i}+S_{\mathrm{AGB}}^{i}+F X_{0}^{i}, \\
m_{\mathrm{GMC}} \frac{d X_{\mathrm{GMC}}^{i}}{d t}=-\frac{m_{\mathrm{GMC}} X_{\mathrm{GMC}}^{i}}{\tau_{\mathrm{GMC}}}+\frac{m_{\mathrm{ICM}} X_{\mathrm{ICM}}^{i}}{\tau_{\mathrm{ICM}}}+S_{\mathrm{GMC}}^{i}-R \mathcal{M} X_{\mathrm{GMC}}^{i},
\end{gathered}
$$

$X_{0}^{i}$ are the isotopic abundances of the infalling gas, and

$$
S_{i}=\int_{M_{1}}^{M_{2}} \frac{R\left(T\left(M_{*}\right)\right)}{\langle M\rangle} P^{i}\left(M_{*}\right) f\left(M_{*}\right) d M_{*},
$$




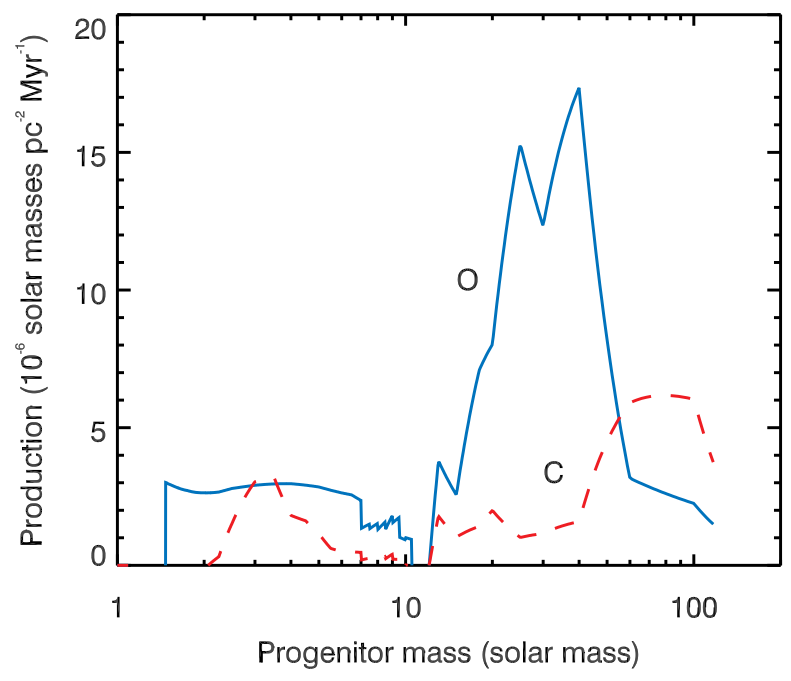

Figure 10. Predicted mass of oxygen (blue, solid) and carbon (red, dashed) from stars vs. progenitor mass for a solar-metallicity population weighted by an IMF with power-law index $\alpha=2.6$

where $P^{i}$ is the production (equal to the nucleosynthetic yield plus the original mass of the isotope). The $\mathrm{C} / \mathrm{O}$ ratio is calculated by summing the appropriate mass fractions divided by the atomic weights. An approximate metallicity is also calcated from the sum of all the isotopes relative to the abundances in the Sun.

I considered nucleosynthesis in the progenitors of AGB stars (1-6.5 $\left.\mathrm{M}_{\odot}\right)$, "super-AGB" stars $\left(6.5-11 \mathrm{M}_{\odot}\right)$, and SN (11$\left.120 \mathrm{M}_{\odot}\right)$. I used the AGB yields from Karakas (2010) for AGB progenitors up to $6.5 \mathrm{M}_{\odot}$. For super-AGB progenitors with masses between 7 and $11 \mathrm{M}_{\odot}$ I used the productions from Dohertv et al. (2014), or Siess (2010) otherwise. For SN yields I adopted the values in Kobavashi et al. (2006) and Kobavashi et al. (2011) for regular SN with energies of $10^{51} \mathrm{erg}$ and their re-run calcalations for the cases of $M_{*}=18 \mathrm{M}_{\odot}$ and $Z=0.004$, and $M_{*}=25$ and $Z=0.02$. These were supplemented with values from Portinari et al. (1998) for progenitor masses of $12,60,100$, and $120 \mathrm{M}_{\odot}$. In order to arrive at a solar $\mathrm{C} / \mathrm{O}$ with reasonable choices of parameters I find it necessary to include yields from the winds of massive stars with $M_{*}>40 \mathrm{M}_{\odot}$ estimated by Portinari et al. (1998) (Table 3 in that work).

The literature for SN and AGB nucleosynthesis is very heterogeneous, with calculations performed for different ranges/values of progenitor masses and metallicities, and it was necessary to interpolate or extrapolate for some values. Specifically, to estimate the production of $6.5 \mathrm{M}_{\odot}$ AGB stars with sub-solar progenitor metallicities I scaled the Karakas (2010) values for sub-solar metallicity $6 \mathrm{M}_{\odot}$ progenitors by the ratio of the $6.5 \mathrm{M}_{\odot}$ to $6 \mathrm{M}_{\odot}$ production for solar-metallicity. For productions from super-AGB stars with progenitor masses outside the caclulated range, I either used the production from the most massive progenitor with the same metallicity, or linearly interpolated between the values for the most massive AGB and least massive super-AGB progenitors with the same metallicity. For a review of the many parameters and uncertainties that enter these calculations see Karakas \& Lattanzio (2014). I linearly interpolated the production onto a grid of 1000 masses over $1-120 \mathrm{M}_{\odot}$ with intervals chosen such that the IMF has equal total mass in each bin. Figure 10 shows the calculated yields (production - initial incorporation) for solar metallicity. These values were then used to evaluate Eqns. A3 and A9.

I searched for the parameter values that best reproduce the observational constraints using Monte-Carlo Markov Chain analysis. The constraints are the age of the Sun, the present mass surface densities of stars, stellar remnants, and total gas at the solar galactocentric radius, solar $\mathrm{C} / \mathrm{O}$, the current metallicity of the ISM (taken to be +0.14 based on the metallicities of M44 and the Hyades), and the mean (-0.05) and intrinsic standard-deviation (0.18 dex) of the metallicity distribution in the solar neighborhood (Gaidos et al. 2014). I adopted standard errors of 0.12 in $\mathrm{C} / \mathrm{O}$ (Caffau et al. 2011) $1.5,1$, and $1 \mathrm{M}_{\odot} \mathrm{pc}^{-2}$ for stars, remnants, and gas, $50 \mathrm{Myr}$ for the absolute age of the Sun, and 0.01 and 0.02 dex in the mean and standard deviation of the present metallicity. I varied the exponential infall timescale, the metallicity of the infalling material, the age of the Galactic disk in the solar annulus, the high-mass IMF index $\alpha$, and the Schmidt-Kennicut index $\beta$.

The simulation is able to adequately reproduce the observed properties of the Galactic disk in the solar neighborhood $\left(\chi^{2}=35\right.$ with $\left.\nu=3\right)$. The most significant deviation is the over-prediction of the present ISM gas mass and underprediction of the current metallicity. The adopted or fit values for the simulation are given in Table 1. The predicted evolution of the SFR, mass surface densities, and the metallicity of the ISM are plotted in Fig. [11, Estimates of the current total star formation rate of the Milky Way cluster around $1.9 \pm 0.4 \mathrm{M}_{\odot} \mathrm{yr}^{-1}$ (Chomiuk \& Povich 2011). Presuming that star formation has an exponential radial distribution with scale length of $3.5 \mathrm{kpc}$ (Wolfire et al. 2003) then the rate at the solar radius will be about $2.7 \pm 0.6 \times 10^{-3} \mathrm{M}_{\odot} \mathrm{Myr}^{-1} \mathrm{pc}^{-2}$. This compares favorably with the predicted value at the present (Fig. 11). 


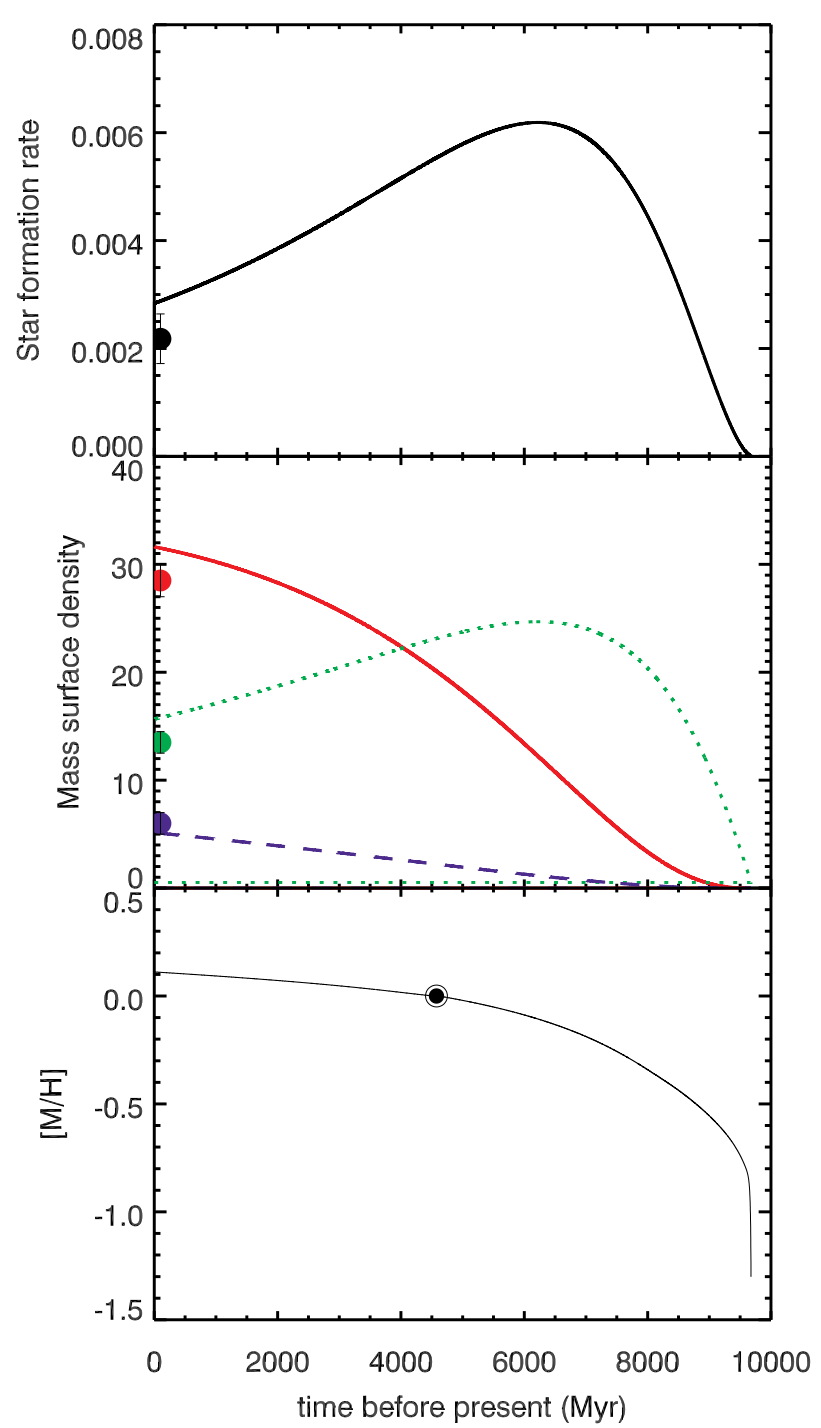

Figure 11. Predictions of the GCE model compared to the Sun and values at present. Top: star formation rate per area compared to the present estimated value. Middle: mass surface densities of stars (solid, red), gas (green, dotted), and stellar remnants (blue, dashed) compared to estimates of present values. (points, Naab \& Ostriker 2006). Bottom: metallicity, here taken to be $[\mathrm{C}+\mathrm{O} / \mathrm{H}]$.

Table 1

GCE Simulation Parameters

\begin{tabular}{|c|c|c|c|c|}
\hline Symbol & Parameter & Value & Units & Reference \\
\hline \multicolumn{5}{|c|}{ Solar Parameters } \\
\hline $\bar{X}^{\mathrm{O}}$ & Solar oxygen & $6.73 \times 10^{-3}$ & - & Caffau et al. (2011) \\
\hline$X^{\mathrm{C}}$ & Solar carbon & $2.73 \times 10^{-3}$ & - & Caffau et al. (2011) \\
\hline \multicolumn{5}{|c|}{ Stellar Parameters } \\
\hline$\alpha$ & IMF index & 2.61 & - & Fit \\
\hline $\mathcal{M}$ & Low mass/high-mass ratio & 2.00 & - & Fit \\
\hline \multicolumn{5}{|c|}{ Galactic Parameters } \\
\hline$m_{*}(T)$ & Present stellar mass density & $28.5 \pm 1.5$ & $\mathrm{M}_{\odot} \mathrm{pc}^{-2}$ & Naab \& Ostriker (2006) \\
\hline$m_{g}(T)$ & Present gas mass density & $13.5 \pm 1$ & $\mathrm{M}_{\odot} \mathrm{pc}^{-2}$ & Naab \& Ostriker (2006) \\
\hline$m_{r}(T)$ & Present remnant mass density & $6 \pm 1$ & $\mathrm{M}_{\odot} \mathrm{pc}^{-2}$ & Naab \& Ostriker (2006) \\
\hline$\tau_{\mathrm{GMC}}$ & GMC gas residence time & 30 & Myr & \\
\hline$\tau_{\mathrm{IMC}}$ & IMC gas residence time & 150 & Myr & \\
\hline$T_{\text {disk }}$ & Age of disk & 9554 & Myr & Fit \\
\hline$\tau_{\text {infall }}$ & Infall e-folding time & 2630 & Myr & Fit \\
\hline$[M / H]_{0}$ & Metallicity of infalling gas & -1.3 & - & Fit \\
\hline$\underline{\beta}$ & SFR rate index & 1.71 & - & Fit \\
\hline
\end{tabular}

